\title{
Exponential Dichotomies for Linear Non-autonomous Functional Differential Equations of Mixed Type
}

\author{
JÖRG HÄRTERICH, BJÖRn SANDSTEDE, AND ARND SCHEEL
}

\begin{abstract}
Functional differential equations with forward and backward delays arise naturally, for instance, in the study of travelling waves in lattice equations and as semi-discretizations of partial differential equations (PDEs) on unbounded domains. Linear functional differential equations of mixed type are typically ill-posed, i.e., there exists, in general, no solution to a given initial condition. We prove that Fredholm properties of these equations imply the existence of exponential dichotomies. Exponential dichotomies can be used in discretized PDEs and in lattice differential equations to construct multi-pulses, to perform Evans-function type calculations, and to justify numerical computations using artificial boundary conditions.
\end{abstract}

\section{INTRODUCTION}

We are interested in linear non-autonomous functional differential equations

$$
v^{\prime}(\xi)=\sum_{j=-m}^{m} A_{j}(\xi) v(\xi+j), \quad \xi \in \mathbb{R}
$$

of mixed type, where $v \in \mathbb{C}^{n}$, and $A_{j}(\xi)$ are continuous functions with values in $\mathbb{C}^{n \times n}$ for $|j| \leq m$. Before we motivate our interest in this equation and list a number of applications that we have in mind, we discuss a few properties of (1.1). The most important feature of (1.1), at least for the purpose of this paper, is that the associated initial-value problem is ill-posed. To make this statement more precise, we should first explain in what sense we want to solve (1.1): We say that a function $v(\xi)$ satisfies (1.1) on an interval $J=[a, b]$, where $a=\infty$ and $b=\infty$ 
are allowed, if $v \in L_{\text {loc }}^{2}\left([a-m, b+m], \mathbb{C}^{n}\right) \cap H_{\text {loc }}^{1}\left([a, b], \mathbb{C}^{n}\right)$, and (1.1) is met in $L_{\text {loc }}^{2}\left([a, b], \mathbb{C}^{n}\right)$. The initial-value problem associated with $(1.1)$ is given by

$$
v^{\prime}(\xi)=\sum_{j=-m}^{m} A_{j}(\xi) v(\xi+j),\left.\quad v\right|_{[-m, m]}=\phi,
$$

where $\phi$ is a given function defined on $[-m, m]$. Unfortunately, for a given function $\phi$, there is in general no solution, in the above sense, to (1.2) on any nontrivial interval that contains $\xi=0$. A simple counterexample (see [15]) is provided by the equation

$$
v^{\prime}(\xi)=v(\xi-1)+v(\xi+1),\left.\quad v\right|_{[-m, m]}=1
$$

with $v \in \mathbb{C}$. The only function that could possibly be a solution of this initialvalue problem is given by $v(\xi)=(-1)^{k}$ for $\xi \in(2 k-1,2 k+1]$ with $k \in \mathbb{N}$; this function, however, is not even continuous. Seeking solutions of the form $v(\xi)=\mathrm{e}^{\lambda \xi}$, we see that the characteristic eigenvalue equation associated with $(1.3)$ is

$$
\lambda=\mathrm{e}^{-\lambda}+\mathrm{e}^{\lambda} .
$$

This equation has solutions $\lambda \in \mathbb{C}$ with $\operatorname{Re} \lambda$ arbitrarily large and also admits solutions for which $-\operatorname{Re} \lambda$ is arbitrarily large. Therefore, the linear equation (1.3) does not generate a semiflow on any space that contains all its eigenfunctions. This explains why the initial-value problem (1.2) associated with (1.1) is ill-posed. In fact, functional differential equations of mixed type behave quite similar to elliptic PDEs when considered as initial-value problems. Note also that solving (1.1) forward or backward in the time variable $\xi$ is equally difficult.

Since we cannot solve (1.2) for all $\phi$, we should therefore find those functions $\phi$ for which a solution to (1.2) exists on either $\mathbb{R}^{+}$or $\mathbb{R}^{-}$. In particular, we would expect to be able to solve the linear autonomous equation (1.3) for $\xi>0$ for any initial condition $\phi$ that is a superposition of eigenfunctions associated with stable eigenvalues (i.e., eigenvalues with negative real part). In fact, the resulting solution should decay to zero exponentially as $\xi \rightarrow \infty$. Analogously, we should be able to solve (1.3) on $\mathbb{R}^{-}$for any initial condition $\phi$ that is a superposition of eigenfunctions associated with unstable eigenvalues (i.e., eigenvalues of positive real part), and the solution should decay exponentially as $\xi \rightarrow-\infty$. Using results from [1] about the characteristic equation, Rustichini [15] proved these assertions for autonomous equations. His result leads naturally to the question how large the closure of all eigenfunctions associated with either stable or unstable eigenvalues is. Indeed, the sum of the resulting closed spaces gives the function space on which we can construct solutions to (1.1) on either $\mathbb{R}^{+}$or $\mathbb{R}^{-}$. The difficulty in determining whether this sum is the entire underlying function space, i.e., whether the set of eigenfunctions is complete, lies in the problem of excluding solutions that decay super-exponentially, so-called small solutions. Verduyn Lunel 
[19] gave conditions that guarantee that the set of eigenfunctions associated with an autonomous functional differential equation is complete.

In this paper, we address the above issues for non-autonomous functional differential equations of mixed type. The obvious difficulty is that the spaces on which we can solve (1.1) forward or backward in time will depend on $\xi$. It is not apriori clear which spaces will replace the unstable and stable eigenspaces that were so useful for autonomous equations. It turns out that the correct notion in the non-autonomous setup are exponential dichotomies. An exponential dichotomy formalizes the idea of solving (1.1) either forward or backward in $\xi$ for initial conditions in certain complementary subspaces even though these subspaces will depend on $\xi$.

To formulate the definition of exponential dichotomies in the present context, it is convenient to introduce the following notation, which we shall use frequently. For a given function $v:[-m, \infty) \rightarrow \mathbb{C}^{n}$, we define $v_{\xi}:[-m, m] \rightarrow \mathbb{C}^{n}$ via $v_{\xi}(\eta):=v(\xi+\eta)$ for $\eta \in[-m, m]$.

Definition 1.1 ([14]). Let $J=[a, b]$ be $\mathbb{R}^{+}, \mathbb{R}^{-}$or $\mathbb{R}$. Equation (1.1) is said to have an exponential dichotomy on the interval $J$ if there exist positive constants $K$ and $\kappa$, and a strongly continuous family of projections $P(\xi)$ : $L^{2}\left([-m, m], \mathbb{C}^{n}\right) \rightarrow L^{2}\left([-m, m], \mathbb{C}^{n}\right)$ such that the following is true for any $\phi \in L^{2}\left([-m, m], \mathbb{C}^{n}\right)$ and $\zeta \in J$.

(i) There exists a unique solution $v$ on $[\zeta, b]$ of (1.1) such that $v_{\zeta}=P(\zeta) \phi$. In addition, $v_{\xi} \in \mathrm{R}(P(\xi))$ and

$$
\left\|v_{\xi}\right\|_{L^{2}\left([-m, m], \mathbb{C}^{n}\right)} \leq K \mathrm{e}^{-\kappa|\xi-\zeta|}\|\phi\|_{L^{2}\left([-m, m], \mathbb{C}^{n}\right)}
$$

for all $\xi \geq \zeta$ with $\xi, \zeta \in J$.

(ii) There exists a unique solution $v$ on $[a, \zeta]$ of (1.1) such that $v_{\zeta}=$ (id $-P(\zeta)) \phi$. In addition, $v_{\xi} \in \mathrm{N}(P(\xi))$ and

$$
\left\|v_{\xi}\right\|_{L^{2}\left([-m, m], \mathbb{C}^{n}\right)} \leq K \mathrm{e}^{-\kappa|\xi-\zeta|}\|\phi\|_{L^{2}\left([-m, m], \mathbb{C}^{n}\right)}
$$

for all $\xi \leq \zeta$ with $\xi, \zeta \in J$.

Exponential dichotomies have been shown to exist in ordinary differential equations [4], parabolic PDEs [7] and delay equations [6], where the unstable subspace $\mathrm{N}(P(\xi))$ is always finite-dimensional, and the initial-value problem is well-posed. In [14], the existence of exponential dichotomies has been established for elliptic PDEs on unbounded domains. Here, both $\mathrm{R}(P(\xi))$ and $\mathrm{N}(P(\xi))$ are infinite-dimensional, and the initial-value problem is ill-posed.

Associated with (1.1) is the operator

$$
\mathcal{L}: H^{1}\left(\mathbb{R}, \mathbb{C}^{n}\right) \rightarrow L^{2}\left(\mathbb{R}, \mathbb{C}^{n}\right), \quad(\mathcal{L} v)(\xi)=\frac{\mathrm{d} v}{\mathrm{~d} \xi}(\xi)-\sum_{j=-m}^{m} A_{j}(\xi) v(\xi+j)
$$


and its formal adjoint $\mathcal{L}^{*}$, defined on the same spaces, given by

$$
\left(\mathcal{L}^{*} w\right)(\xi)=-\frac{\mathrm{d} w}{\mathrm{~d} \xi}(\xi)-\sum_{j=-m}^{m} A_{j}^{*}(\xi-j) w(\xi-j) .
$$

We need the following weak uniqueness assumption.

Hypothesis 1.1. If $v$ is in the null space of $\mathcal{L}$ or the adjoint operator $\mathcal{L}^{*}$ such that $\left.v\right|_{\left[\xi_{0}-m, \xi_{0}+m\right]}=0$ for some $\xi_{0}$, then $v$ vanishes identically.

Our main result is the following theorem.

Theorem 1.1. If $\mathcal{L}$ is a Fredholm operator and if Hypothesis 1.1 is met, then (1.1) has exponential dichotomies on $\mathbb{R}^{+}$and on $\mathbb{R}^{-}$.

A weaker but perhaps more explicit version of the above theorem is the following statement.

Theorem 1.2. If (1.1) is asymptotically hyperbolic (see Definition 2.1 below) and if neither $\operatorname{det} A_{m}(\xi)$ nor $\operatorname{det} A_{-m}(\xi)$ vanish on any open interval, then (1.1) has exponential dichotomies on $\mathbb{R}^{+}$and on $\mathbb{R}^{-}$. [12].

Analogous results have been shown, independently and simultaneously, in

The existence of exponential dichotomies on $\mathbb{R}^{+}$and $\mathbb{R}^{-}$has a number of consequences: for instance, the null space and the orthogonal complement of the range of $\mathcal{L}$ are isomorphic to the spaces $\mathrm{R}\left(P_{+}(0)\right) \cap \mathrm{N}\left(P_{-}(0)\right)$ and $\left(\mathrm{R}\left(P_{+}(0)\right)+\right.$ $\left.\mathrm{N}\left(P_{-}(0)\right)\right)^{\perp}$, respectively. In addition, it is possible to characterize the Fredholm index of $\mathcal{L}$ by the difference of relative Morse indices. We refer to [18] for details.

Lastly, we motivate why functional differential equations are interesting and outline some applications of exponential dichotomies that we intend to pursue in future work. Linear non-autonomous functional differential equations of mixed type arise in many different problems. We may, for instance, be interested in travelling waves of lattice differential equations

$$
\partial_{t} u_{k}=\sum_{j=-m}^{m} f_{j}\left(u_{k+j}\right), \quad k \in \mathbb{Z},
$$

where $u_{j}=u_{j}(t)$ for $j \in \mathbb{Z}$. A travelling-wave solution is a function $\varphi(\xi)$ such that, for some wave speed $c \in \mathbb{R}$, we have $u_{k}(t)=\varphi(k+c t)$ for $t \in \mathbb{R}$ and $k \in \mathbb{Z}$. Upon substituting this expression for $u_{k}$ into the above lattice equation, we obtain

$$
c \varphi^{\prime}(\xi)=\sum_{j=-m}^{m} f_{j}(\varphi(\xi+j)), \quad \xi \in \mathbb{R}
$$


where we set $\xi=k+c t$. The linearization about the wave $\varphi(\xi)$ is then given by

$$
c v^{\prime}(\xi)=\sum_{j=-m}^{m} \mathrm{D} f_{j}(\varphi(\xi+j)) v(\xi+j)=: \sum_{j=-m}^{m} A_{j}(\xi) v(\xi+j)
$$

which is of the form (1.1) provided the wave speed $c$ does not vanish. Note that, if $c=0$, then the above equation is a difference equation. A second example are semi-discretizations of parabolic PDEs such as

$$
\partial_{t} u=D \partial_{x}^{2} u+f(u), \quad u \in \mathbb{R}^{n}, x \in \mathbb{R}
$$

that admit travelling-wave solutions which connect two, possibly different, homogeneous equilibria. Since such equations are often too complicated to allow for a complete analysis, numerical methods have to be employed to compute travelling waves and to continue them in parameter space. An important question is then to which extent the numerical scheme is able to reproduce travelling waves of the original PDE and whether the stability properties of the wave are retained upon discretizing. We shall investigate these issues in a simplified setting: instead of considering a fully discrete numerical scheme, we may study semi-discretizations, i.e., equations where only the spatial derivatives are replaced by finite difference approximations. The resulting lattice equations are of the form

$$
\partial_{t} u(x, t)=\sum_{j=-m}^{m} \alpha_{j} u(x+j h, t)+f(u(x, t)),
$$

where the coefficients $\alpha_{j}$ may depend on the mesh size $h$. Let $\xi:=(x+c t) / h$, then a travelling wave of the form $u(x, t)=\varphi((x+c t) / h)$ satisfies the nonlinear functional differential equation

$$
\frac{c}{h} \varphi^{\prime}(\xi)=\sum_{j=-m}^{m} \alpha_{j} \varphi(\xi+j)+f(\varphi(\xi)) .
$$

We assume that we have found a solution $\varphi(\xi)$ of this equation and consider the linearization

$$
\frac{c}{h} v^{\prime}(\xi)=\sum_{j=-m}^{m} \alpha_{j} v(\xi+j)+\mathrm{D} f(\varphi(\xi)) v(\xi)
$$

about the wave. If the wave speed $c \neq 0$ is not zero, we obtain a functional differential equation of mixed type as in our first example. Exponential dichotomies provide a useful tool to investigate such equations. In a nutshell (see [18] for a more comprehensive discussion), exponential dichotomies allow for a much 


\section{JÖrg HÄrTERICH. BJÖrn SANDSTEDE, AND ARnd SCHEEl}

more refined perturbation analysis compared with, for instance, Fredholm properties. One example where dichotomies are useful is in providing correct choices of boundary conditions so that (1.1) truncated to an interval $(-L, L)$ with $L \gg 1$ is well-posed (see [10] and the references therein). Dichotomies are also useful in the construction of Evans functions that can be used to investigate linear stability of travelling waves (see, for instance, $[2,18,17]$ and references therein). Lastly, exponential dichotomies can be used to construct new patterns, such as periodic or multi-hump waves, from a given travelling wave by using, for example, Lin's method $[9,16]$. Some of the above issues will be investigated in more detail in a forthcoming article.

This paper is organized as follows. In Section 2, we formulate (1.1) as an evolution problem and introduce several operators relevant to that formulation. In Section 3, we then show the existence of exponential dichotomies for the corresponding autonomous equation. The analysis is similar to the one given in [15] and uses additional results from [19]. Lastly, in Section 4, we consider the non-autonomous equation for which we prove the existence of exponential dichotomies following the strategy in [18].

Acknowledgments. J. Härterich was supported by the Deutsche Forschungsgemeinschaft under grant Ha 3008/1-1. B. Sandstede was partially supported by the National Science Foundation under grant DMS-9971703 and by an Alfred P. Sloan Research Fellowship.

\section{THE OPERATORS $\mathcal{L}$ AND $\mathcal{T}$}

In this section we describe two linear operators that can be associated with the linear functional differential equation

$$
v^{\prime}(\xi)=\sum_{j=-m}^{m} A_{j}(\xi) v(\xi+j)
$$

and show how they are related.

2.1. The operators $\mathcal{L}$ and $\mathcal{L}^{*}$. We define the closed and densely defined linear operator

$$
\begin{gathered}
\mathcal{L}: \mathrm{D}(\mathcal{L})=H^{1}\left(\mathbb{R}, \mathbb{C}^{n}\right) \rightarrow L^{2}\left(\mathbb{R}, \mathbb{C}^{n}\right) \\
(\mathcal{L} v)(\xi)=\frac{\mathrm{d} v}{\mathrm{~d} \xi}(\xi)-\sum_{j=-m}^{m} A_{j}(\xi) v(\xi+j)
\end{gathered}
$$

associated with equation (2.1). The adjoint operator $\mathcal{L}^{*}$ of $\mathcal{L}$ is given by

$$
\left(\mathcal{L}^{*} w\right)(\xi)=-\frac{\mathrm{d} w}{\mathrm{~d} \xi}(\xi)-\sum_{j=-m}^{m} A_{j}^{*}(\xi-j) w(\xi-j) .
$$


It is easily checked that

$$
\int_{-\infty}^{\infty}(\mathcal{L} v)(\xi) \cdot w(\xi) \mathrm{d} \xi=\int_{-\infty}^{\infty} v(\xi) \cdot\left(\mathcal{L}^{*} w\right)(\xi) \mathrm{d} \xi
$$

for all $v, w \in H^{1}\left(\mathbb{R}, \mathbb{C}^{n}\right)$, where we denote the scalar product in $\mathbb{C}^{n}$ by $a \cdot b=$ $\sum_{k=1}^{n} a_{k} \overline{b_{k}}$ for $a, b \in \mathbb{C}^{n}$. The operator $\mathcal{L}$ has particularly nice properties if the coefficients $A_{j}$ satisfy a certain hyperbolicity condition.

Definition 2.1. The linear functional differential equation (2.1) is called asymptotically constant if the $\operatorname{limits}^{ \pm}:=\lim _{\xi \rightarrow \pm \infty} A_{j}(\xi)$ exist for all $j$. The equation is called asymptotically hyperbolic if it is asymptotically constant and if the characteristic equations

$$
\operatorname{det} \Delta_{ \pm}(\mu):=\operatorname{det}\left(\sum_{j=-m}^{m} A_{j}^{ \pm} \mathrm{e}^{\mu j}-\mu \mathrm{id}\right)=0
$$

associated with the limiting equations at $\xi= \pm \infty$ have no solutions $\mu$ on the imaginary axis. If the coefficients do not depend on $\xi$, then we call (2.1) hyperbolic if $\operatorname{det} \Delta(\mu)$ has no purely imaginary zeros $\mu$.

The following result is due to Mallet-Paret [11].

Proposition 2.1 ([11]). If $\mathcal{L}$ is asymptotically hyperbolic, then $\mathcal{L}$ is a Fredholm operator.

2.2. The operator $\mathcal{T}$. A different way of viewing (2.1) is to write it in the form

$$
\frac{\mathrm{d} V}{\mathrm{~d} \xi}(\xi)=\mathcal{A}(\xi) V(\xi)
$$

where, for each fixed $\xi \in \mathbb{R}$, we define

$$
\mathcal{A}(\xi): \quad \mathrm{D}(\mathcal{A}(\xi))=Y^{1} \longrightarrow Y, \quad\left(\begin{array}{l}
\phi \\
a
\end{array}\right) \longmapsto\left(\begin{array}{c}
\frac{\mathrm{d} \phi}{\mathrm{d} \eta} \\
A_{0}(\xi) a+\sum_{1 \leq|j| \leq m} A_{j}(\xi) \phi(j)
\end{array}\right)
$$

with

$$
\begin{aligned}
Y & :=L^{2}\left([-m, m], \mathbb{C}^{n}\right) \times \mathbb{C}^{n} \\
Y^{1} & :=\left\{(\phi, a) \in H^{1}\left([-m, m], \mathbb{C}^{n}\right) \times \mathbb{C}^{n} ; \phi(0)=a\right\} .
\end{aligned}
$$

The space $Y^{1}$ is well defined since $H^{1}\left([-m, m], \mathbb{C}^{n}\right)$ is embedded in $C^{0}\left([-m, m], \mathbb{C}^{n}\right)$. Note also that $Y^{1}$ is dense in $Y$ since the set of $C^{1}$-functions 


\section{Jörg HÄrTERICH. BJÖrn SANDSTEDE, AND ARnd SCHEEl}

$\phi$ with $\phi(0)=a$ is dense in the set of step functions in the $L^{2}$-norm. The operator $\mathcal{A}(\xi)$ has domain $Y^{1}$ and is closed for any fixed $\xi$. In the case of constant coefficients, we write $\mathcal{A}_{0}$ instead of $\mathcal{A}(\xi)$.

Before we define the operator $\mathcal{T}$, we state the following lemma that we use below to define the domain of $\mathcal{T}$.

Lemma 2.1. Using the notation $I=[-m, m]$ and $(\xi, \eta) \in \mathbb{R} \times I$, we have that $L^{2}\left(\mathbb{R}, L^{2}\left(I, \mathbb{C}^{n}\right)\right)=L^{2}\left(\mathbb{R} \times I, \mathbb{C}^{n}\right)$. Furthermore, there is a constant $C$ with the following property. If $\phi \in L^{2}\left(\mathbb{R} \times I, \mathbb{C}^{n}\right)$ such that the weak derivative $\left(\partial_{\xi}-\partial_{\eta}\right) \phi \in L^{2}\left(\mathbb{R} \times I, \mathbb{C}^{n}\right)$ exists, then $\phi(\cdot, k) \in L^{2}\left(\mathbb{R}, \mathbb{C}^{n}\right)$ for every fixed $k \in I$ and $\phi(0, \cdot) \in L^{2}\left(I, \mathbb{C}^{n}\right)$; in addition, we have

$\|\phi(0, \cdot)\|_{L^{2}\left(I, \mathbb{C}^{n}\right)}+\|\phi(\cdot, k)\|_{L^{2}\left(\mathbb{R}, \mathbb{C}^{n}\right)} \leq C\left(\|\phi\|_{L^{2}\left(\mathbb{R} \times I, \mathbb{C}^{n}\right)}+\left\|\left(\partial_{\xi}-\partial_{\eta}\right) \phi\right\|_{L^{2}\left(\mathbb{R} \times I, \mathbb{C}^{n}\right)}\right)$.

Proof. The identity $L^{2}\left(\mathbb{R}, L^{2}\left(I, \mathbb{C}^{n}\right)\right)=L^{2}\left(\mathbb{R} \times I, \mathbb{C}^{n}\right)$ is a consequence of Fubini's theorem. Upon introducing the coordinates $(\tilde{\xi}, \tilde{\eta})=(\xi+\eta, \eta) \in \mathbb{R} \times I$, we see that $\phi(\xi, \eta) \in L^{2}\left(\mathbb{R} \times I, \mathbb{C}^{n}\right)$ with $\left(\partial_{\xi}-\partial_{\eta}\right) \phi(\xi, \eta) \in L^{2}\left(\mathbb{R} \times I, \mathbb{C}^{n}\right)$ if, and only if, $\tilde{\phi}(\tilde{\xi}, \tilde{\eta}):=\phi(\tilde{\xi}-\tilde{\eta}, \tilde{\eta}) \in L^{2}\left(\mathbb{R}, H^{1}\left(I, \mathbb{C}^{n}\right)\right)$. In particular, $\tilde{\phi} \in$ $L^{2}\left(\mathbb{R}, C^{0}\left(I, \mathbb{C}^{n}\right)\right)$, and we conclude that $\tilde{\phi}(\cdot, k) \in L^{2}\left(\mathbb{R}, \mathbb{C}^{n}\right)$ for every fixed $k \in I$. Hence, $\phi(\xi, k)=\tilde{\phi}(\xi+k, k) \in L^{2}\left(\mathbb{R}, \mathbb{C}^{n}\right)$ for fixed $k$. Lastly, for any such $\phi$, we also have that $\phi(0, \eta)=\tilde{\phi}(\eta, \eta)$ exists for almost every $\eta \in I$. It is straightforward to see that the $L^{2}$-norm of $\tilde{\phi}(\eta, \eta)$ can be bounded by the $L^{2}(\mathbb{R} \times I)$-norms of $\phi$ and $\left(\partial_{\xi}-\partial_{\eta}\right) \phi$ upon using the coordinates $(\tilde{\xi}, \tilde{\eta})$.

Associated with (2.2) is the operator

$$
\begin{aligned}
\mathcal{T}: \quad L^{2}(\mathbb{R}, Y) & \longrightarrow L^{2}(\mathbb{R}, Y), \quad V \longmapsto \frac{\mathrm{d} V}{\mathrm{~d} \xi}(\cdot)-\mathcal{A}(\cdot) V(\cdot) \\
(\phi, a) & \longmapsto\left(\frac{\mathrm{d} \phi}{\mathrm{d} \xi}-\frac{\mathrm{d} \phi}{\mathrm{d} \eta}, \frac{\mathrm{d} a}{\mathrm{~d} \xi}(\xi)-A_{0}(\xi) a(\xi)-\sum_{1 \leq|j| \leq m} A_{j}(\xi)[\phi(\xi)](j)\right)
\end{aligned}
$$

which is considered as an unbounded operator on $L^{2}(\mathbb{R}, Y)$ with domain

$$
\begin{aligned}
\mathrm{D}(\mathcal{T})=\left\{(\phi, a) \in L^{2}(\mathbb{R}, Y) ;\right. & \left(\partial_{\xi}-\partial_{\eta}\right) \phi \in L^{2}\left(\mathbb{R} \times I, \mathbb{C}^{n}\right), \\
& \left.a \in H^{1}\left(\mathbb{R}, \mathbb{C}^{n}\right),[\phi(\xi)](0)=a(\xi) \forall \xi\right\}
\end{aligned}
$$

where we use the notation $I=[-m, m]$. Note that $\mathrm{D}(\mathcal{T})$ is well-defined owing to Lemma 2.1. Using again Lemma 2.1, it is not difficult to prove that $\mathcal{T}$ is closed and densely defined.

Note that we have $L^{2}\left(\mathbb{R}, Y^{1}\right) \cap H^{1}(\mathbb{R}, Y) \subset \mathrm{D}(\mathcal{T})$. It is tempting to take $L^{2}\left(\mathbb{R}, Y^{1}\right) \cap H^{1}(\mathbb{R}, Y)$ as the domain of $\mathcal{T}$. The operator $\mathcal{T}$ is, however, not closed if considered with this domain. 

related.

The following lemma shows how the null spaces of the operators $\mathcal{L}$ and $\mathcal{T}$ are

Lemma 2.2. If a function $v \in H^{1}\left(\mathbb{R}, \mathbb{C}^{n}\right)$ satisfies $\mathcal{L} v=0$, then $V(\xi):=$ $\left(\left.v\right|_{[\xi-m, \xi+m]}, v(\xi)\right)$ satisfies $V \in \mathrm{D}(\mathcal{T})$ and $\mathcal{T} V=0$. Conversely, if $V=(\phi, a) \in$ $\mathrm{D}(\mathcal{T})$ satisfies $\mathcal{T} V=0$, then $a \in H^{1}\left(\mathbb{R}, \mathbb{C}^{n}\right)$ satisfies $\mathcal{L} a=0$. In particular, $\mathrm{N}(\mathcal{L}) \cong \mathrm{N}(\mathcal{T})$.

Proof. If $v \in H^{1}\left(\mathbb{R}, \mathbb{C}^{n}\right)$ is a solution to $\mathcal{L} v=0$, then $v$ is in fact of class $C^{\infty}$. To see this, it suffices to show that $v \in C^{k}\left([-\ell, \ell], \mathbb{C}^{n}\right)$ for arbitrarily large numbers $k$ and $\ell$. By the Sobolev Embedding Theorem, $v$ is in $C^{0}$ on the interval $[-\ell-m k, \ell+m k]$. Since $v$ satisfies (2.1), it is $C^{1}$ on the smaller interval $[-\ell-(k-1) m, \ell+(k-1) m]$. Inductively one can then show that $v$ is indeed of class $C^{k}$ on $[-\ell, \ell]$. As a consequence, $V(\xi):=\left(v_{\xi}, v(\xi)\right)$ is a classical solution of (2.2). It remains to check that $V$ is in the domain of $\mathcal{T}$. Since $v \in H^{1}\left(\mathbb{R}, \mathbb{C}^{n}\right)$, we know that

$$
\begin{aligned}
\|V\|_{L^{2}\left(\mathbb{R}, Y^{1}\right)} & =\int_{\mathbb{R}} \int_{-m}^{m}\left(\left|v_{\xi}(\eta)\right|^{2}+\left|\partial_{\eta} v_{\xi}(\eta)\right|^{2}\right) \mathrm{d} \eta \mathrm{d} \xi+\|v\|_{H^{1}\left(\mathbb{R}, \mathbb{C}^{n}\right)} \\
& =\int_{\mathbb{R}} \int_{-m}^{m}\left(|v(\xi+\eta)|^{2}+\left|v^{\prime}(\xi+\eta)\right|^{2}\right) \mathrm{d} \eta \mathrm{d} \xi+\|v\|_{H^{1}\left(\mathbb{R}, \mathbb{C}^{n}\right)} \\
& =(2 m+1)\|v\|_{H^{1}\left(\mathbb{R}, \mathbb{C}^{n}\right)},
\end{aligned}
$$

and we conclude that $V$ is in $L^{2}\left(\mathbb{R}, Y^{1}\right)$. Note that $v \in H^{1}\left(\mathbb{R}, \mathbb{C}^{n}\right)$ is in the domain of the derivative which is the generator of the shift semigroup on $L^{2}\left(\mathbb{R}, \mathbb{C}^{n}\right)$. Therefore,

$$
\frac{\mathrm{d}}{\mathrm{d} \xi} v(\xi+\cdot)=\frac{\mathrm{d}}{\mathrm{d} \eta} v(\xi+\cdot) .
$$

Hence $V$ is in $H^{1}(\mathbb{R}, Y)$ and therefore indeed in $L^{2}\left(\mathbb{R}, Y^{1}\right) \times H^{1}(\mathbb{R}, Y) \subset \mathrm{D}(\mathcal{T})$.

To show the other direction, assume that $V=(\phi, a) \in \mathrm{D}(\mathcal{T})$ satisfies $\mathcal{T} V=$ 0 . It follows from the definition of $\mathrm{D}(\mathcal{T})$ that $v(\xi)=a(\xi)=[\phi(\xi)](0)$ is well-defined and $v \in H^{1}\left(\mathbb{R}, \mathbb{C}^{n}\right)$. It remains to show that $\left.a\right|_{[\xi-m, \xi+m]}=\phi(\xi)$, i.e., $[\phi(\xi+\eta)](0)=[\phi(\xi)](\eta)$. As in Lemma 2.1, we use the coordinates $(\tilde{\xi}, \tilde{\eta})=(\xi+\eta, \eta) \in \mathbb{R} \times I$ and set $\tilde{\phi}(\tilde{\xi}, \tilde{\eta}):=[\phi(\tilde{\xi}-\tilde{\eta})](\tilde{\eta})$. From $\frac{\mathrm{d}}{\mathrm{d} \xi} \phi=\frac{\mathrm{d}}{\mathrm{d} \eta} \phi$, we conclude that $\tilde{\phi}(\tilde{\xi}, \tilde{\eta})=\tilde{\phi}(\tilde{\xi}, 0)$ for almost every $\tilde{\xi}$, and therefore for every $\tilde{\xi}$ as $[\phi(\xi)](0)$ is continuous. Hence, $[\phi(\xi-\eta)](\eta)=[\phi(\xi)](0)$ for every $\xi$ and $\eta$, and we conclude that $[\phi(\xi+\eta)](0)=[\phi(\xi)](\eta)$.

2.3. The adjoint operator $\mathcal{T}^{*}$. We denote by $\langle\cdot, \cdot\rangle$ the inner product

$$
\left\langle\left(\begin{array}{l}
\phi \\
a
\end{array}\right),\left(\begin{array}{l}
\psi \\
b
\end{array}\right)\right\rangle:=\int_{-\infty}^{\infty} \int_{-m}^{m} \phi(\xi, \eta) \cdot \psi(\xi, \eta) \mathrm{d} \eta \mathrm{d} \xi+\int_{-\infty}^{\infty} a(\xi) \cdot b(\xi) \mathrm{d} \xi
$$

on $L^{2}(\mathbb{R}, Y)$. 
1090 JÖrg HÄrterich. BJÖrn SANDSTEDE, AND ARnd SCHEEL

Remark 2.1. Let $j$ be an integer with $-m \leq j<m$. If $\psi \in$ $L^{2}\left(\mathbb{R} \times[j, j+1], \mathbb{C}^{n}\right)$ with weak derivative $\left(\partial_{\xi}-\partial_{\eta}\right) \psi \in L^{2}\left(\mathbb{R} \times(j, j+1), \mathbb{C}^{n}\right)$, then $\psi(\cdot, j)$ and $\psi(\cdot, j+1)$ are in $L^{2}\left(\mathbb{R}, \mathbb{C}^{n}\right)$ by Lemma 2.1 . We use the notation $\psi(\cdot, j+):=\psi(\cdot, j)$ and $\psi(\cdot,(j+1)-):=\psi(\cdot, j+1)$. For convenience, we also define $\psi(\cdot, m+)=\psi(\cdot,-m-)=0$.

Lemma 2.3. The adjoint operator $\mathcal{T}^{*}$ is given by

$$
\begin{aligned}
\mathcal{T}^{*}: L^{2}(\mathbb{R}, Y) & \longrightarrow L^{2}(\mathbb{R}, Y), \\
(\psi, b) & \longmapsto\left(-\frac{\mathrm{d} \psi}{\mathrm{d} \xi}+\frac{\mathrm{d} \psi}{\mathrm{d} \eta},-\frac{\mathrm{d} b}{\mathrm{~d} \xi}-A_{0}^{*}(\xi) b+\psi(\cdot, 0-)-\psi(\cdot, 0+)\right)
\end{aligned}
$$

with

$$
\begin{aligned}
\mathrm{D}\left(\mathcal{T}^{*}\right)=\left\{(\psi, b) \in L^{2}(\mathbb{R}, Y) ;\left(\partial_{\xi}-\partial_{\eta}\right) \psi \in L^{2}\left(\mathbb{R} \times(j, j+1), \mathbb{C}^{n}\right)\right. & \\
& \forall j \text { with }-m \leq j<m, \\
b \in H^{1}\left(\mathbb{R}, \mathbb{C}^{n}\right), \psi(\xi, j-)-\psi(\xi, j+)= & A_{j}^{*}(\xi) b(\xi) \\
& \forall \xi \text { and } 0<|j| \leq m\}
\end{aligned}
$$

(see Remark 2.1 for the notation). Furthermore, $\mathcal{T}^{*}(\psi, b)=0$ if, and only if, $\mathcal{L}^{*} b=0$.

Proof. The domain $\mathrm{D}\left(\mathcal{T}^{*}\right)$ of the adjoint operator $\mathcal{T}^{*}$ is given by

$$
\begin{aligned}
\mathrm{D}\left(\mathcal{T}^{*}\right)=\left\{(\psi, b) \in L^{2}(\mathbb{R}, Y) ;\right. & \\
\exists\left(\psi_{*}, b_{*}\right) \in L^{2}(\mathbb{R}, Y):\langle\mathcal{T}(\phi, a),(\psi, b)\rangle=\left\langle(\phi, a),\left(\psi_{*}, b_{*}\right)\right\rangle & \forall(\phi, a) \in \mathrm{D}(\mathcal{T})\},
\end{aligned}
$$

in which case $\mathcal{T}^{*}(\psi, b):=\left(\psi_{*}, b_{*}\right)$. Thus, we consider the equation

$$
\begin{aligned}
\int_{-\infty}^{\infty} \int_{-m}^{m}\left(\frac{\mathrm{d} \phi}{\mathrm{d} \xi}-\frac{\mathrm{d} \phi}{\mathrm{d} \eta}\right) \cdot \psi \mathrm{d} \eta \mathrm{d} \xi & \\
+\int_{-\infty}^{\infty}\left(\frac{\mathrm{d} a}{\mathrm{~d} \xi}-A_{0}(\xi) a-\right. & \left.\sum_{j \neq 0} A_{j}(\xi) \phi(\xi, j)\right) \cdot b \mathrm{~d} \xi \\
& =\int_{-\infty}^{\infty} \int_{-m}^{m} \phi \cdot \psi_{*} \mathrm{~d} \eta \mathrm{d} \xi+\int_{-\infty}^{\infty} a \cdot b_{*} \mathrm{~d} \xi
\end{aligned}
$$


Upon setting $a=0$, so that $\phi(\xi, 0)=0$, we obtain

$$
\begin{aligned}
\int_{-\infty}^{\infty} \int_{-m}^{m}\left(\frac{\mathrm{d} \phi}{\mathrm{d} \xi}-\frac{\mathrm{d} \phi}{\mathrm{d} \eta}\right) \cdot \psi \mathrm{d} \eta \mathrm{d} \xi-\int_{-\infty}^{\infty} \sum_{j \neq 0} A_{j}(\xi) \phi(\xi, j) \cdot b \mathrm{~d} \xi \\
=\int_{-\infty}^{\infty} \int_{-m}^{m} \phi \cdot \psi_{*} \mathrm{~d} \eta \mathrm{d} \xi
\end{aligned}
$$

If we restrict to test functions $\phi$ with $\phi(\xi, j)=0$ for all integers $j$, we see that $\psi_{*}=\left(\partial_{\eta}-\partial_{\xi}\right) \psi$ in $L^{2}\left(\mathbb{R} \times(j, j+1), \mathbb{C}^{n}\right)$ for all $j$ with $-m \leq j<m$. Note that this defines $\psi_{*}$ uniquely in $L^{2}\left(\mathbb{R} \times[-m, m], \mathbb{C}^{n}\right)$. Using the notation introduced in Remark 2.1 and considering arbitrary test functions $\phi$ with $\phi(\xi, 0)=0$, we obtain

$$
\begin{aligned}
& \int_{-\infty}^{\infty} \int_{-m}^{m}\left(\frac{\mathrm{d} \phi}{\mathrm{d} \xi}-\frac{\mathrm{d} \phi}{\mathrm{d} \eta}\right) \cdot \psi \mathrm{d} \eta \mathrm{d} \xi \\
& \quad=\int_{-\infty}^{\infty} \int_{-m}^{m} \phi \cdot \psi_{*} \mathrm{~d} \eta \mathrm{d} \xi+\int_{-m}^{m} \sum_{j \neq 0}^{m} \phi(\xi, j) \cdot(\psi(\xi, j-)-\psi(\xi, j+)) \mathrm{d} \eta
\end{aligned}
$$

and conclude that (2.6) is met for all $\phi$ with $\phi(\xi, 0)=0$ provided

$$
\psi(\cdot, j-)-\psi(\cdot, j+)=A_{j}^{*}(\cdot) b(\cdot)
$$

in $L^{2}\left(\mathbb{R}, \mathbb{C}^{n}\right)$ for all $j \neq 0$. We return to $(2.5)$ which, based on the results established above, reduces to

$$
\begin{aligned}
\int_{-m}^{m} \phi(\xi, 0) \cdot(\psi(\xi, 0-)- & \psi(\xi, 0+)) \mathrm{d} \eta \\
& +\int_{-\infty}^{\infty}\left(\frac{\mathrm{d} a}{\mathrm{~d} \xi}-A_{0}(\xi) a\right) \cdot b \mathrm{~d} \xi=\int_{-\infty}^{\infty} a \cdot b_{*} \mathrm{~d} \xi
\end{aligned}
$$

and therefore to

$$
\int_{-\infty}^{\infty} \frac{\mathrm{d} a}{\mathrm{~d} \xi} \cdot b \mathrm{~d} \xi=\int_{-\infty}^{\infty} a \cdot\left(b_{*}+A_{0}^{*}(\xi) b-\psi(\xi, 0-)+\psi(\xi, 0+)\right) \mathrm{d} \xi
$$

for all $a \in H^{1}\left(\mathbb{R}, \mathbb{C}^{n}\right)$, since $\phi(\cdot, 0)=a$ for every $(\phi, a) \in \mathrm{D}(\mathcal{T})$. This shows that $b \in H^{1}\left(\mathbb{R}, \mathbb{C}^{n}\right)$ and

$$
b_{*}=-\frac{\mathrm{d} b}{\mathrm{~d} \xi}-A_{0}^{*} b+\psi(\cdot, 0-)-\psi(\cdot, 0+)
$$


Lastly, the proof that the null spaces of $\mathcal{T}^{*}$ and $\mathcal{L}^{*}$ are isomorphic is quite analogous to the proof of Lemma 2.2. The following argument is the key. Suppose that $(\psi, b) \in \mathrm{D}\left(\mathcal{T}^{*}\right)$ with $\mathcal{T}^{*}(\psi, b)=0$. Using that $\left(\partial_{\xi}-\partial_{\eta}\right) \psi=0$ and that $\psi(\xi, j-)=\psi(\xi, j+)+A_{j}^{*}(\xi) b(\xi)$ for $j>0$ with $\psi(\xi, m-)=A_{m}^{*}(\xi) b(\xi)$, it follows inductively that

$$
\psi(\xi, j-)=\sum_{k=j}^{m} A_{k}^{*}(\xi-k+j) b(\xi-k+j)
$$

for $j>0$. Hence,

$$
\psi(\xi, 0+)=\psi(\xi-1,1-)=\sum_{j=0}^{m} A_{j}^{*}(\xi-j) b(\xi-j) .
$$

Similarly, we obtain

$$
\psi(\xi, 0-)=\psi(\xi+1,-1+)=-\sum_{j=0}^{-m} A_{j}^{*}(\xi-j) b(\xi-j)
$$

and therefore

$\frac{\mathrm{d} b}{\mathrm{~d} \xi}=-A_{0}^{*}(\xi) b+\psi(\cdot, 0-)-\psi(\cdot, 0+)=-A_{0}^{*}(\xi) b-\sum_{j=-m}^{m} A_{j}^{*}(\xi-j) b(\xi-j)$.

We omit the remaining details.

As a consequence, $\mathcal{T}$ is Fredholm whenever $\mathcal{L}$ is.

Lemma 2.4. If $\mathcal{L}$ is Fredholm with index $i$, then $\mathcal{T}$ is Fredholm with the same index $i$.

Proof. On account of Lemma 2.2 and Lemma 2.3, we have $\operatorname{dim} \mathrm{N}(\mathcal{L})=$ $\operatorname{dim} \mathrm{N}(\mathcal{T})$ and $\operatorname{dim} \mathrm{N}\left(\mathcal{L}^{*}\right)=\operatorname{dim} \mathrm{N}\left(\mathcal{T}^{*}\right)$. To show that the range of $\mathcal{T}$ is closed, assume that $\mathcal{T}\left(\phi_{n}, \alpha_{n}\right) \rightarrow(\psi, \beta)$ is a convergent sequence in $\mathrm{R}(\mathcal{T})$. Define $v_{n}(\xi):=\phi_{n}(\xi)(0)=\alpha_{n}(\xi)$ which gives a sequence $\left(v_{n}\right)_{n \in \mathbb{N}}$ in $H^{1}\left(\mathbb{R}, \mathbb{C}^{n}\right)$. The sequence $\left(\mathcal{L} v_{n}\right)$ converges in $L^{2}\left(\mathbb{R}, \mathbb{C}^{n}\right)$ to $\beta$. Since $\mathrm{R}(\mathcal{L})$ is closed, we know that $\mathcal{L} v_{n} \rightarrow \mathcal{L} v_{\infty}$ for some $v_{\infty} \in H^{1}\left(\mathbb{R}, \mathbb{C}^{n}\right)$. Lemma 2.2 implies that $(\psi, \beta)=$ $\mathcal{T}\left(\phi_{\infty}, \alpha_{\infty}\right)$ for $\phi_{\infty}(\xi):=\left.v_{\infty}\right|_{[\xi-m, \xi+m]}$ and $\alpha_{\infty}:=v_{\infty}$. Thus, $R(\mathcal{T})$ is closed, and we conclude that $\mathrm{R}\left(\mathcal{T}^{*}\right)$ is also closed [8, Theorem IV.5.13]. In addition, we then have $\operatorname{codim} R(\mathcal{L})=\operatorname{codim} R(\mathcal{T})$ since

$$
\operatorname{codim} \mathrm{R}(\mathcal{L})=\operatorname{dim} \mathrm{N}\left(\mathcal{L}^{*}\right)=\operatorname{dim} \mathrm{N}\left(\mathcal{T}^{*}\right)=\operatorname{codim} \mathrm{R}(\mathcal{T})
$$

by the arguments at the beginning of the proof. 


\section{The CONSTANT-COEFFICIENT OPERATOR $\mathcal{A}_{0}$}

For given matrices $A_{j}$ with $|j| \leq m$, consider the constant-coefficient operator

$$
\mathcal{A}_{0}: \quad Y \rightarrow Y, \quad\left(\begin{array}{l}
\phi \\
a
\end{array}\right) \longmapsto\left(\begin{array}{c}
\frac{\mathrm{d} \phi}{\mathrm{d} \eta} \\
A_{0} a+\sum_{1 \leq|j| \leq m} A_{j} \phi(j)
\end{array}\right)
$$

which is densely defined with domain $\mathrm{D}\left(\mathcal{A}_{0}\right)=Y^{1}$ (see (2.3)). The characteristic equation associated with $\mathcal{A}_{0}$ is $\operatorname{det} \Delta(\mu)=0$ where

$$
\Delta(\mu):=\sum_{j=-m}^{m} A_{j} \mathrm{e}^{j \mu}-\mu \mathrm{id} .
$$

We are interested in proving the existence of exponential dichotomies for the equation

$$
\frac{\mathrm{d} V}{\mathrm{~d} \xi}=\mathcal{A}_{0} V
$$

since this equation with constant coefficients serves as a reference equation for the more general case that we consider in Section 4 below. To prove the existence of exponential dichotomies for equations with constant coefficients, we first show that $\mathcal{A}_{0}$ has only point spectrum and provide estimates for its resolvent. Afterwards, we establish in Section 3.2 the completeness of eigenfunctions and proceed then in Section 3.3 with the construction of exponential dichotomies in a fashion similar to Rustichini [15] who proved the existence of dichotomies for slightly different operators in $C^{0}\left([-m, m], \mathbb{C}^{n}\right)$.

3.1. Spectrum, and resolvent estimates. We begin by establishing that the spectrum of $\mathcal{A}_{0}$ consists entirely of eigenvalues.

Lemma 3.1. The operator $\mathcal{A}_{0}$ has only point spectrum. Moreover, $\mu \in \mathbb{C}$ is in the spectrum of $\mathcal{A}_{0}$ if, and only if, $\operatorname{det} \Delta(\mu)=0$. If $\mu$ is an eigenvalue, the corresponding eigenfunction is of the form $(\phi, a)$ with $a \in \mathbb{C}^{n}$ and $\phi(\eta)=a \mathrm{e}^{\eta \mu}$.

Proof. To determine the spectrum and the resolvent of $\mathcal{A}_{0}$ we have to discuss the equation

$$
\left(\mathcal{A}_{0}-\mu\right)\left(\begin{array}{l}
\phi \\
a
\end{array}\right)=\left(\begin{array}{l}
\psi \\
b
\end{array}\right)
$$

where $\psi \in L^{2}\left([-m, m], \mathbb{C}^{n}\right)$ and $b \in \mathbb{C}^{n}$. This equation is equivalent to

$$
\begin{gathered}
\frac{\mathrm{d} \phi}{\mathrm{d} \eta}-\mu \phi=\psi \\
\left(A_{0}-\mu\right) a+\sum_{1 \leq|j| \leq m} A_{j} \phi(j)=b .
\end{gathered}
$$


1094 JÖRG HäRTERICH. BJÖRn SANDSTEDE, AND ARND SCHEEL

Solving the first equation by the variations-of-constants formula shows that

$$
\phi(\eta)=\mathrm{e}^{\eta \mu} \phi(0)+\int_{0}^{\eta} \mathrm{e}^{(\eta-\sigma) \mu} \psi(\sigma) \mathrm{d} \sigma .
$$

Substituting this expression for $\phi$ into the second equation and exploiting that $\phi(0)=a$, we get

$$
\Delta(\mu) a=b-\sum_{j \neq 0} A_{j} \int_{0}^{j} \mathrm{e}^{(j-\sigma) \mu} \psi(\sigma) \mathrm{d} \sigma .
$$

Therefore, for any $\mu$ with $\operatorname{det} \Delta(\mu) \neq 0$, i.e., for any $\mu$ that is not in the point spectrum of $\mathcal{A}_{0}$, the resolvent of $\mathcal{A}_{0}$ is given by

$$
\begin{array}{r}
\phi(\eta)=\mathrm{e}^{\eta \mu} \Delta(\mu)^{-1}\left(b-\sum_{j \neq 0} A_{j} \int_{0}^{j} \mathrm{e}^{(j-\sigma) \mu} \psi(\sigma) \mathrm{d} \sigma\right) \\
+\int_{0}^{\eta} \mathrm{e}^{(\eta-\sigma) \mu} \psi(\sigma) \mathrm{d} \sigma \\
a=\Delta(\mu)^{-1}\left(b-\sum_{j \neq 0} A_{j} \int_{0}^{j} \mathrm{e}^{(j-\sigma) \mu} \psi(\sigma) \mathrm{d} \sigma\right) .
\end{array}
$$

For later use, we derive estimates for the resolvent of $\mathcal{A}_{0}$. Rustichini [15] proved similar estimates for linear operators associated with constant-coefficient equations considered in $C^{0}\left([-m, m], \mathbb{C}^{n}\right)$.

Lemma 3.2. Fix a constant $\kappa>0$. There is then a constant $M>0$ such that, for any $\mu$ with $\operatorname{det} \Delta(\mu) \neq 0$ and $|\operatorname{Re} \mu|>\kappa$, we have

$$
\left\|\left(\mathcal{A}_{0}-\mu\right)^{-1}\right\|_{L(Y)} \leq M\left(\frac{\mathrm{e}^{m|\operatorname{Re} \mu|}}{|\operatorname{Re} \mu|}+\left\|\Delta(\mu)^{-1}\right\| \frac{\mathrm{e}^{2 m|\operatorname{Re} \mu|}}{|\operatorname{Re} \mu|}\right) .
$$

Proof. We have shown that the equation

$$
\left(\mathcal{A}_{0}-\mu\right)\left(\begin{array}{l}
\phi \\
a
\end{array}\right)=\left(\begin{array}{l}
\psi \\
b
\end{array}\right) \in Y
$$

has the solution

$$
\begin{aligned}
\phi(\eta) & =\mathrm{e}^{\eta \mu} \Delta(\mu)^{-1}\left(b-\sum_{j \neq 0} A_{j} \int_{0}^{j} \mathrm{e}^{(j-\sigma) \mu} \psi(\sigma) \mathrm{d} \sigma\right)+\int_{0}^{\eta} \mathrm{e}^{(\eta-\sigma) \mu} \psi(\sigma) \mathrm{d} \sigma \\
a & =\Delta(\mu)^{-1}\left(b-\sum_{j \neq 0} A_{j} \int_{0}^{j} \mathrm{e}^{(j-\sigma) \mu} \psi(\sigma) \mathrm{d} \sigma\right)
\end{aligned}
$$


whenever $\operatorname{det} \Delta(\mu) \neq 0$ with $\Delta(\mu)=\sum_{j=-m}^{m} A_{j} \mathrm{e}^{j \mu}-\mu$ id. Note that

$$
\left|\int_{0}^{y} \mathrm{e}^{2(y-\sigma) \operatorname{Re} \mu} \mathrm{d} \sigma\right|^{1 / 2} \leq \frac{\mathrm{e}^{|y \operatorname{Re} \mu|}}{\sqrt{2|\operatorname{Re} \mu|}} .
$$

Using this inequality in combination with Hölder's inequality, we find that

$$
\begin{aligned}
|a| & \leq\left\|\Delta(\mu)^{-1}\right\|\left(|b|+\sum_{j \neq 0}\left\|A_{j}\right\| \frac{\mathrm{e}^{|j \operatorname{Re} \mu|}}{\sqrt{2|\operatorname{Re} \mu|}}\|\psi\|_{L^{2}}\right) \\
& \leq\left\|\Delta(\mu)^{-1}\right\|\left(|b|+M_{1} \frac{\mathrm{e}^{m|\operatorname{Re} \mu|}}{\sqrt{2|\operatorname{Re} \mu|}}\|\psi\|_{L^{2}}\right) \\
& \leq\left\|\Delta(\mu)^{-1}\right\|\left(1+M_{1} \frac{\mathrm{e}^{m|\operatorname{Re} \mu|}}{\sqrt{2|\operatorname{Re} \mu|}}\right)\left\|\left(\begin{array}{l}
\psi \\
b
\end{array}\right)\right\|_{Y}
\end{aligned}
$$

where

$$
M_{1}:=2 m \max _{-m \leq j \leq m}\left\|A_{j}\right\| .
$$

The same estimate appears when we bound the $L^{2}$-norm of $\phi$ :

$$
\begin{aligned}
& \|\phi\|_{L^{2}} \leq\left\|\mathrm{e}^{\eta \mu}\right\|_{L^{2}}\left\|\Delta(\mu)^{-1}\right\|\left(1+M_{1} \frac{\mathrm{e}^{m|\operatorname{Re} \mu|}}{\sqrt{2|\operatorname{Re} \mu|}}\right)\left\|\left(\begin{array}{l}
\psi \\
b
\end{array}\right)\right\|_{Y}+\left\|\frac{\mathrm{e}^{|\eta \operatorname{Re} \mu|}}{\sqrt{2 \mid \operatorname{Re} \mu} \|}\right\|_{L^{2}}\|\psi\|_{L^{2}}
\end{aligned}
$$

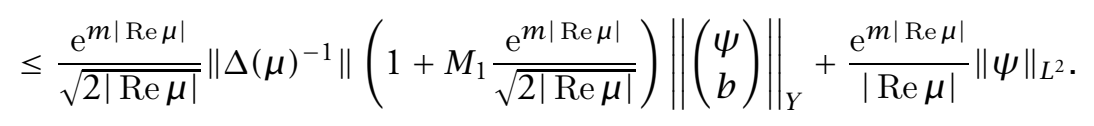

For $|\operatorname{Re} \mu|>\kappa$, this implies the desired inequality.

Using results of Bellman and Cooke [1], Rustichini demonstrated the following statement on the location of zeros of $\Delta(\mu)$.

Lemma 3.3 ([15, Lemma 3.2]). Assume that $\operatorname{det}\left(A_{-m} A_{m}\right) \neq 0$, then the following is true.

(i) For any $\kappa>0$, there exists a constant $M(\kappa)$ such that $|\operatorname{Im} \mu| \leq M(\kappa)$ for any zero $\mu$ of $\Delta(\mu)$ with $|\operatorname{Re} \mu| \leq \kappa$. In particular, if there are no zeros of $\Delta(\mu)$ on the imaginary axis, then there is a strip $\{\mu ;|\operatorname{Re} \mu| \leq \kappa\}$ that contains no eigenvalues of $\mathcal{A}_{0}$.

(ii) There exists a positive constant $\tilde{\kappa}$ such that all zeros of $\Delta$ are contained in the union $W_{+} \cup W_{-}$where

$$
W_{ \pm}:=\left\{\mu \in \mathbb{C} ;|\operatorname{Re} \mu|>\kappa,\left|\operatorname{Re}\left(\mu \pm \frac{1}{m} \log \mu\right)\right| \leq \widetilde{\kappa}\right\} .
$$


(iii) There exists a constant $C>0$ such that

$$
\left\|\Delta(\mu)^{-1}\right\| \leq C \mathrm{e}^{-m|\operatorname{Re} \mu|}
$$

along the curves $|\operatorname{Re}(\mu \pm(1 / m) \log \mu)|=\widetilde{\kappa}$, that is, along curves where $|\mu| \mathrm{e}^{ \pm m|\operatorname{Re} \mu|}$ is constant.

3.2. Completeness. An important property, which is not at all obvious for non-selfadjoint operators such as $\mathcal{A}_{0}$, is completeness, i.e., the property that the closure of the linear space spanned by the generalized eigenfunctions of $\mathcal{A}_{0}$ is the entire underlying function space $Y$. We demonstrate that $\mathcal{A}_{0}$ is complete provided that the matrices $A_{-m}$ and $A_{m}$ are not singular.

Theorem 3.1. Consider the operator $\mathcal{A}_{0}$ defined in (3.1). Assume that $\mathcal{A}_{0}$ has no spectrum on the imaginary axis and that $\operatorname{det}\left(A_{-m} A_{m}\right) \neq 0$. Let $\overline{E^{\mathrm{u}}}$ be the closure in $Y$ of the sum of generalized eigenspaces to all eigenvalues with positive real part. Similarly, let $\overline{E^{\mathrm{s}}}$ be the closure of the sum of the generalized eigenspace corresponding to eigenvalues with negative real part. We then have $\overline{E^{\mathrm{u}}} \oplus \overline{E^{\mathrm{s}}}=Y$.

Remark 3.1. An analogous statement holds if the spectrum has a center part, i.e., in the situation that there are eigenvalues on the imaginary axis.

Our proof of Theorem 3.1 is based on a characterization of completeness given by Verduyn Lunel in [19] (see Lemma 3.8 below). We begin by recalling some facts from complex analysis.

Definition 3.1. Let $X$ be a complex Banach space. An entire function $F$ : $\mathbb{C} \rightarrow X$ is said to be of exponential type $E(F)$ if

$$
\limsup _{r \rightarrow \infty} \frac{1}{r} \log \max _{0 \leq \theta \leq 2 \pi}\left\|F\left(r \mathrm{e}^{\mathrm{i} \theta}\right)\right\|=: E(F)<\infty
$$

exists.

In the following proposition, we summarize some properties of entire functions of exponential type.

Lemma 3.4. Let $F_{1}, F_{2}: \mathbb{C} \rightarrow \mathbb{C}$ be entire functions of exponential type which are polynomially bounded in the closed right half-plane $\operatorname{Re} \mu \geq 0$. We then have $E\left(F_{1} F_{2}\right)=E\left(F_{1}\right)+E\left(F_{2}\right)$. If the quotient $F_{1} / F_{2}$ is an entire function, then $E\left(F_{1} / F_{2}\right)=$ $E\left(F_{1}\right)-E\left(F_{2}\right)$.

Note that the same holds for entire functions which are bounded in the left half-plane. This symmetry will allow us later to relax some conditions. The following lemma will prove useful below.

Lemma 3.5. If $F: \mathbb{C} \rightarrow \mathbb{C}$ is an entire function of exponential type that satisfies

$$
\underset{r \rightarrow \infty}{\limsup } \frac{1}{r} \log |F( \pm \mathrm{i} r)|=0 \quad \text { and } \quad|F(\mu)| \leq M \quad \text { for } \mu \in \mathbb{R},
$$


then $F$ is constant.

Proof. The assertion is a consequence of [3, Thm. 6.2.4], which is a theorem by Duffin and Schaeffer [5], applied separately to $F(\mu)$ and $F(-\mu)$ with $\mu$ restricted to the upper half-plane in conjunction with Liouville's Theorem.

Using the identity

$$
\Delta(\mu)^{-1}=\frac{1}{\operatorname{det} \Delta(\mu)} \operatorname{cof} \Delta(\mu),
$$

where $\operatorname{cof} \Delta(\mu)$ is the matrix of cofactors, we can rewrite the solution of the equation

$$
\left(\mathcal{A}_{0}-\mu\right)\left(\begin{array}{l}
\phi \\
a
\end{array}\right)=\left(\begin{array}{l}
\psi \\
b
\end{array}\right)
$$

given in (3.2) as

$$
\begin{gathered}
\phi(\eta)=\frac{\mathrm{e}^{\eta \mu}}{\operatorname{det} \Delta(\mu)} \operatorname{cof} \Delta(\mu)\left(b-\sum_{j \neq 0} A_{j} \int_{0}^{j} \mathrm{e}^{(j-\sigma) \mu} \psi(\sigma) \mathrm{d} \sigma\right) \\
+\int_{0}^{\eta} \mathrm{e}^{(\eta-\sigma) \mu} \psi(\sigma) \mathrm{d} \sigma \\
a=\frac{\operatorname{cof} \Delta(\mu)}{\operatorname{det} \Delta(\mu)}\left(b-\sum_{j \neq 0} A_{j} \int_{0}^{j} \mathrm{e}^{(j-\sigma) \mu} \psi(\sigma) \mathrm{d} \sigma\right) .
\end{gathered}
$$

It is not hard to see that some of the functions involved in the above expression are entire functions of exponential type.

Lemma 3.6. The exponential type of $\operatorname{det} \Delta(\mu)$ is $m n$ if, and only if, $\operatorname{det} A_{-m} \neq$ 0 or $\operatorname{det} A_{m} \neq 0$. In this case the exponential type of each entry of the cofactor matrix $\operatorname{cof} \Delta(\mu)$ is $m(n-1)$.

Moreover, if $\operatorname{det} A_{-m} \neq 0$, then $\mathrm{e}^{-m n \mu} \operatorname{det} \Delta(\mu)$ is of exponential type $2 m n$ and bounded in the right half-plane, while the entries of $\mathrm{e}^{-m(n-1) \mu} \operatorname{cof} \Delta(\mu)$ are bounded in the right half-plane and have exponential type $2 m(n-1)$.

Similarly, if $\operatorname{det} A_{m} \neq 0$, then $\mathrm{e}^{m n \mu} \operatorname{det} \Delta(\mu)$ is of exponential type $2 m n$ and bounded in the left half-plane, while the entries of $\mathrm{e}^{m(n-1) \mu} \operatorname{cof} \Delta(\mu)$ are bounded in the left half-plane and have exponential type $2 m(n-1)$.

Proof. The term det $\Delta(\mu)$ is a linear combination of terms of the form $\mu^{q} \mathrm{e}^{j \mu}$ where $0 \leq q \leq n$ and $-m n \leq j \leq m n$. This implies that the exponential type of $\operatorname{det} \Delta(\mu)$ cannot be greater than $m n$. The coefficient of $\mathrm{e}^{m n \mu}$ is $\operatorname{det} A_{m}$, while the coefficient of $\mathrm{e}^{-m n \mu}$ is $\operatorname{det} A_{-m}$. If at least one of those coefficients is nonzero, then the definition of exponential type with $\mu$ restricted to the real line shows that $E(\operatorname{det} \Delta)=m n$. On the other hand, the exponential type is strictly less than 
$m n$ if both $\operatorname{det} A_{-m}$ and $\operatorname{det} A_{m}$ vanish. Similar arguments apply to the entries of $\operatorname{cof} \Delta(\mu)$. Note that, if $\operatorname{det} A_{ \pm m} \neq 0$, then each sub-determinant of $A_{ \pm m}$ does not vanish either. The remaining estimates can be obtained by completely analogous arguments.

In view of Lemma 3.5 it is also important to control the behavior of the resolvent along the imaginary axis.

Lemma 3.7. We have the following asymptotic behavior on the imaginary axis:

$$
\begin{array}{rlrl}
\lim _{r \rightarrow \infty} \frac{\operatorname{det} \Delta( \pm \mathrm{i} r)}{r^{n}} & =1 & \\
\lim _{r \rightarrow \infty} \frac{(\operatorname{cof} \Delta( \pm \mathrm{i} r))_{k k}}{r^{n-1}} & =1 & & \text { for } k=1,2, \ldots, n \\
\lim _{r \rightarrow \infty} \frac{(\operatorname{cof} \Delta( \pm \mathrm{i} r))_{k l}}{r^{n-2}} & =(-1)^{k+l} & & \text { for } k \neq l .
\end{array}
$$

Proof. These relations are simple consequences of the fact that along the imaginary axis the polynomial terms dominate the exponential terms.

We use the following characterization of non-completeness.

Lemma 3.8 ([19, Lemma 3.2]). If B : $X \rightarrow X$ is an unbounded operator with meromorphic resolvent, then the system of eigenfunctions and generalized eigenfunctions is not complete if, and only if, there exists a $y^{*} \in X^{*}$ with $y^{*} \neq 0$ such that the function

$$
\mu \longmapsto\left\langle y^{*},(B-\mu)^{-1} x\right\rangle
$$

is entire for every fixed $x \in X$.

We will show that no such $y^{*}$ exists in our situation. The explicit form of the resolvent $\left(\mathcal{A}_{0}-\mu\right)^{-1}$ shows that it is indeed a meromorphic function so that Lemma 3.8 applies to $\mathcal{A}_{0}$.

Proof of Theorem 3.1. Assume that the system of eigenvectors and generalized eigenvectors of $\mathcal{A}_{0}$ is not complete. Applying Lemma 3.8 and the Riesz representation theorem to (3.3), we see that there are $(\phi, a) \in Y$ such that, for every fixed $(\psi, b) \in Y$, the function

$$
\begin{aligned}
& \left\langle\left(\begin{array}{l}
\phi \\
a
\end{array}\right),\left(\mathcal{A}_{0}-\mu\right)^{-1}\left(\begin{array}{l}
\psi \\
b
\end{array}\right)\right\rangle \\
& =a \cdot \frac{1}{\operatorname{det} \Delta(\mu)} \operatorname{cof} \Delta(\mu)\left(b-\sum_{j \neq 0} A_{j} \int_{0}^{j} \mathrm{e}^{(j-\sigma) \mu} \psi(\sigma) \mathrm{d} \sigma\right) \\
& \quad+\int_{-m}^{m} \phi(\eta) \cdot \frac{1}{\operatorname{det} \Delta(\mu)} \operatorname{cof} \Delta(\mu)\left(\mathrm{e}^{\eta \mu} b-\sum_{j \neq 0} A_{j} \int_{0}^{j} \mathrm{e}^{(\eta+j-\sigma) \mu} \psi(\sigma) \mathrm{d} \sigma\right) \mathrm{d} \eta+
\end{aligned}
$$




$$
+\int_{-m}^{m} \phi(\eta) \cdot\left(\int_{0}^{\eta} \mathrm{e}^{(\eta-\sigma) \mu} \psi(\sigma) \mathrm{d} \sigma\right) \mathrm{d} \eta
$$

is entire. In particular, this is true for $\psi=0$ for which the above expression reduces to

$$
\begin{aligned}
\left\langle\left(\begin{array}{l}
\phi \\
a
\end{array}\right),\left(\mathcal{A}_{0}-\mu\right)^{-1}\left(\begin{array}{l}
0 \\
b
\end{array}\right)\right\rangle=a & \frac{1}{\operatorname{det} \Delta(\mu)} \operatorname{cof} \Delta(\mu) b \\
& +\int_{-m}^{m} \phi(\eta) \cdot \frac{1}{\operatorname{det} \Delta(\mu)} \operatorname{cof} \Delta(\mu) \mathrm{e}^{\eta \mu} b \mathrm{~d} \eta
\end{aligned}
$$

If the right-hand side defines an entire function for all $b \in \mathbb{C}^{n}$, then each component $F_{k}(\mu)$ of

$$
F(\mu):=a \cdot \frac{\operatorname{cof} \Delta(\mu)}{\operatorname{det} \Delta(\mu)}+\int_{-m}^{m} \phi(\eta) \cdot \frac{\operatorname{cof} \Delta(\mu)}{\operatorname{det} \Delta(\mu)} \mathrm{e}^{\eta \mu} \mathrm{d} \eta
$$

is an entire function. We prove that this implies that $\phi=0$ which leads to a contradiction.

We first show that each $F_{k}(\mu)$ satisfies the assumptions of Lemma 3.5 with $c=0$. Note that, since $\operatorname{det} A_{-m} \neq 0$, by Lemma 3.6 both the numerator and the denominator of $\mathrm{e}^{-m n \mu} F_{k}(\mu)$ are entire functions of exponential type $2 m n$ which are bounded in the right half-plane. Assuming that $F_{k}$ is itself entire, we can conclude from Lemma 3.4 that $F_{k}$ is of exponential type 0 . Regarding the behavior of $F_{k}$ along the imaginary axis, Lemma 3.7 shows that $F_{k}(\mathrm{i} r$ ) converges to 0 for $r \rightarrow \pm \infty$. This implies directly the weaker statement

$$
\limsup _{r \rightarrow \infty} \frac{1}{r} \log \left|F_{k}( \pm \mathrm{i} r)\right| \leq 0
$$

which is used in Lemma 3.5. The last hypothesis that needs to be checked is the boundedness on the real axis. Since by assumption $F$ is an entire function, we only need to be concerned about the behavior for $\mu \rightarrow \pm \infty$. Since $\operatorname{det} A_{-m} \neq 0$ and $\operatorname{det} A_{m} \neq 0$, we conclude that

$$
|\operatorname{det} \Delta(\mu)| \geq C \mathrm{e}^{m n|\mu|}
$$

for some constant $C$ and $|\mu|$ sufficiently large. On the other hand, cof $\Delta(\mu)$ is of exponential type $m(n-1)$, whence we can estimate

$$
\left|\int_{-m}^{m} \phi(\eta) \cdot \operatorname{cof} \Delta(\mu) \mathrm{e}^{\eta \mu} b \mathrm{~d} \eta\right| \leq\left|\int_{-m}^{m} \phi(\eta) \mathrm{e}^{m(n-1)|\mu|} \mathrm{e}^{m|\mu|} b \mathrm{~d} \eta\right|
$$

which implies that $F_{k}(\mu)$ is uniformly bounded for large $|\mu|$. 
Hence, Lemma 3.5 implies that each $F_{k}$ is constant. Using the behavior along the imaginary axis, we conclude immediately that this constant is zero so that

$$
F_{k}(\mu) \operatorname{det} \Delta(\mu)=a \cdot \operatorname{cof} \Delta(\mu)+\int_{-m}^{m} \phi(\eta) \cdot \operatorname{cof} \Delta(\mu) \mathrm{e}^{\eta \mu} \mathrm{d} \eta=0 .
$$

This implies that

$$
-a=\int_{-m}^{m} \phi(\eta) \mathrm{e}^{\eta \mu} \mathrm{d} \eta=\int_{0}^{2 m} \phi(\eta-m) \mathrm{e}^{m \mu} \mathrm{e}^{\eta \mu} \mathrm{d} \eta
$$

is a constant independent of $\mu$. In other words, if we extend $\phi$ to $\mathbb{R}$ by setting $\phi(\eta)=0$ for $|\eta|>m$, then the constant function $-a$ would be the Laplace transform of the function $\phi(\eta-m) \mathrm{e}^{m \mu}$ which is in $L^{2}$ and has compact support. However, as the Laplace of a function with compact support, $-a$ would have to be integrable along each line $\operatorname{Re} \mu=$ const. This would imply $a=0$, and by inverse Laplace transform $\phi=0$. Therefore, by Lemma 3.8, the operator $\mathcal{A}_{0}$ has a complete system of eigenvectors and generalized eigenvectors.

3.3. Exponential dichotomies. We are now in a position to establish the existence of exponential dichotomies for the equation

$$
\frac{\mathrm{d} V}{\mathrm{~d} \xi}=\mathcal{A}_{0} V
$$

with constant coefficients. We assume that $\mathcal{A}_{0}$ is hyperbolic, i.e., that $\Delta(\mu) \neq 0$ for all $\mu \in \mathrm{i} \mathbb{R}$. Recall from Lemma 3.3 that the distance from the spectrum of $\mathcal{A}_{0}$ to the imaginary axis is strictly positive. Let $\overline{E^{\mathrm{s}}}$ and $\overline{E^{\mathrm{u}}}$ be the closure in $Y$ of the generalized eigenspaces associated with all eigenvalues of $\mathcal{A}_{0}$ that have negative and positive real part, respectively.

Proposition 3.1. Assume that $\operatorname{det}\left(A_{-m} A_{m}\right) \neq 0$, and choose $\kappa>0$ such that $\kappa$ is smaller than the distance from the spectrum of $\mathcal{A}_{0}$ to the imaginary axis. There exists then a strongly continuous semigroup $\Phi^{\mathrm{s}}(\xi): \overline{E^{\mathrm{s}}} \rightarrow \overline{E^{\mathrm{s}}}$ defined for $\xi \geq 0$ and a constant $K$ such that $\Phi^{\mathrm{s}}(0)=\mathrm{id}$ and

$$
\left\|\Phi^{\mathrm{s}}(\xi)\right\|_{L(Y)} \leq K \mathrm{e}^{-\kappa \xi} .
$$

For any $V_{0} \in \overline{E^{\mathrm{s}}} \cap Y^{1}$, the function $V(\xi)=\Phi^{\mathrm{s}}(\xi) V_{0}$ is differentiable for $\xi>0$ and satisfies (3.4) with $V(0)=V_{0}$. Analogous statements hold for $\Phi^{\mathrm{u}}(\xi): \overline{E^{\mathrm{u}}} \rightarrow \overline{E^{\mathrm{u}}}$ defined for $\xi \leq 0$.

Note that, for $V_{0} \in \overline{E^{\mathrm{s}}}$, the function $V(\xi)=\Phi^{\mathrm{s}}(\xi) V_{0}$ is a mild solution of (3.4), i.e., it satisfies the integral equation

$$
V(\xi)=V_{0}+\int_{0}^{\xi} \mathcal{A}_{0} V(\zeta) \mathrm{d} \zeta
$$

for all $\xi \geq 0$. 
Proof. It suffices to prove the statement for $\Phi^{\mathrm{s}}(\xi)$. We begin by constructing semigroups on the subspace $E^{\mathrm{s}}$ which is defined as the sum of all generalized eigenspaces of eigenvalues $\mu$ of $\mathcal{A}_{0}$ with $\operatorname{Re} \mu<0$.

We construct the semigroup $\Phi^{\mathrm{s}}$ via an integral representation. We begin by choosing curves $\Gamma_{1}$ and $\Gamma_{2}$ in $\mathbb{C}$ by

$$
\begin{aligned}
& \Gamma_{1}:=\left\{\mu \in \mathbb{C}: \operatorname{Re}\left(\mu+\frac{1}{m} \log \mu\right)=\tilde{\kappa}, \operatorname{Im} \mu<0, \operatorname{Re} \mu \leq-\kappa\right\} \\
& \Gamma_{2}:=\left\{\mu \in \mathbb{C}: \operatorname{Re}\left(\mu+\frac{1}{m} \log \mu\right)=\tilde{\kappa}, \operatorname{Im} \mu>0, \operatorname{Re} \mu \leq-\kappa\right\}
\end{aligned}
$$

where $\widetilde{\kappa}$ has been defined in Lemma 3.3. Note that $\Gamma_{1}$ and $\Gamma_{2}$ can be parametrized by $\mu=x+\mathrm{i} y(x)$ where $y^{\prime}(x)=\mathrm{O}\left(\mathrm{e}^{-m x}\right)$ as $x \rightarrow-\infty$ (see [15, p. 140]). Lastly, the curve $\Gamma_{3}$ joins $\Gamma_{1}$ and $\Gamma_{2}$ along the line $\operatorname{Re} \mu=-\kappa$. By Lemma 3.3, the curve $\Gamma:=\Gamma_{1} \cup \Gamma_{2} \cup \Gamma_{3}$ lies to the right of the negative part of the spectrum.

For $V_{0} \in E^{\mathrm{s}}$ and $\xi_{0}>2 m$, we have

$$
\begin{aligned}
& \left\|\frac{1}{2 \pi \mathrm{i}} \int_{\Gamma_{1}} \mathrm{e}^{\mu \xi_{0}}\left(\mathcal{A}_{0}-\mu\right)^{-1} V_{0} \mathrm{~d} \mu\right\|_{Y} \\
& \quad \leq \frac{1}{2 \pi} \int_{\Gamma_{1}} \mathrm{e}^{\xi_{0} \operatorname{Re} \mu}\left\|\left(\mathcal{A}_{0}-\mu\right)^{-1}\right\| \mathrm{d} \mu\left\|V_{0}\right\|_{Y} \\
& \quad \leq \frac{1}{2 \pi} \int_{\Gamma_{1}} \mathrm{e}^{\xi_{0} \operatorname{Re} \mu} M\left(\frac{\mathrm{e}^{m|\operatorname{Re} \mu|}}{|\operatorname{Re} \mu|}+C \mathrm{e}^{-m|\operatorname{Re} \mu|} \frac{\mathrm{e}^{2 m|\operatorname{Re} \mu|}}{|\operatorname{Re} \mu|}\right) \mathrm{d} \mu\left\|V_{0}\right\|_{Y} \\
& \quad \leq \frac{1}{2 \pi} \int_{-\infty}^{0} \mathrm{e}^{\xi_{0} x} \mathrm{e}^{-m x} M \frac{1+C}{|x|} \mathrm{e}^{-m x} \mathrm{~d} x\left\|V_{0}\right\|_{Y} \\
& \quad=K\left(\xi_{0}\right)\left\|V_{0}\right\|_{Y}
\end{aligned}
$$

where we used the above parametrization of $\Gamma_{1}$ to evaluate the line integral. Note that the last integral converges for $\xi_{0}>2 \mathrm{~m}$. Since the same calculation applies to the integral along $\Gamma_{2}$, we can define an operator $\Phi^{\mathrm{s}}(\xi)$ on $E^{\mathrm{s}}$ for $\xi \geq \xi_{0}>2 m$ by

$$
\Phi^{\mathrm{s}}(\xi) V_{0}:=\frac{1}{2 \pi \mathrm{i}} \int_{\Gamma} \mathrm{e}^{\mu \xi}\left(\mathcal{A}_{0}-\mu\right)^{-1} V_{0} \mathrm{~d} \mu .
$$

In particular, for $\xi \geq \xi_{0}>2 m$, we have

$$
\left\|\Phi^{\mathrm{s}}(\xi) V_{0}\right\|_{Y} \leq\left\|\frac{1}{2 \pi \mathrm{i}} \int_{\Gamma} \mathrm{e}^{\mu \xi}\left(\mathcal{A}_{0}-\mu\right)^{-1} V_{0} \mathrm{~d} \mu\right\| \leq \mathrm{e}^{-\kappa \xi} K\left(\xi_{0}\right) \mathrm{e}^{\kappa \xi_{0}}\left\|V_{0}\right\|_{Y}
$$

for some $K\left(\xi_{0}\right) \geq 1$, which gives exponential decay for $\xi \geq \xi_{0}>2 m$. Note that, for $V_{0} \in E^{\mathrm{s}} \cap Y^{1}$, the function $V(\xi)=\Phi^{\mathrm{s}}(\xi) V_{0}$ satisfies (3.4) for $\xi>2 m$ with $V(0)=V_{0}($ see $[13])$. 
1102 JÖrg HÄrterich. BJÖrn SANDSTEDE, AND ARnd SCHEEL

Next, we define a filtration of $E^{\mathrm{s}}$ by finite-dimensional generalized eigenspaces $E_{j}^{\mathrm{s}} \subset Y^{1}$ with $j \in \mathbb{N}$ (see [15]). For any $V_{0} \in E_{j}^{\mathrm{s}}$, we can readily solve (3.4) on $\mathbb{R}^{+}$and get a solution $V(\xi)$ that is differentiable with values in $Y$. On the interval $\left[\xi_{0}, \infty\right)$, the solution $V(\xi)$ coincides with $\Phi^{\mathrm{s}}(\xi) V_{0}$ as defined in (3.5). In particular, with $V_{0}=(\phi, \phi(0))$ and $V(\xi)=\left(v_{\xi}, v(\xi)\right)$, the function $v(\xi)$ is a solution of

$$
v^{\prime}(\xi)=\sum_{j=-m}^{m} A_{j} v(\xi+j)
$$

for $\xi \geq 0$ with $\left.v\right|_{[-m, m]}=\phi$. Hence, using (3.6), we obtain the $L^{2}$-estimate

$$
\|v\|_{L^{2}([m+\varepsilon, 3 m+\varepsilon])} \leq K(2 m+\varepsilon)\|\phi\|_{L^{2}}
$$

for any $0<\varepsilon<1 / 2$. Our goal is to estimate the solution $v$ in $L^{2}([m, m+\varepsilon])$ in terms of the $L^{2}$-norm of the initial condition $\phi$. Let $C_{1}$ be a bound for the norms of the matrices $A_{j}$. Using that $v$ satisfies (3.7), we then get the $H^{1}$-estimate

$$
\|v\|_{H^{1}([m+\varepsilon, m+2 \varepsilon])} \leq m C_{1}(1+K(2 m+\varepsilon))\|\phi\|_{L^{2}},
$$

and finally

$$
|v(m+\varepsilon)| \leq C_{2}(1+K(2 m+\varepsilon))\|\phi\|_{L^{2}}
$$

by Sobolev's embedding theorem for some $C_{2}$ that may depend on $\varepsilon$. Multiplying (3.7) by $v(\xi)$, we obtain

$$
v^{\prime}(\xi) \cdot v(\xi)=A_{0} v(\xi) \cdot v(\xi)+\sum_{j \neq 0} A_{j} v(\xi+j) \cdot v(\xi)
$$

and therefore

$\frac{1}{2} \frac{\mathrm{d}}{\mathrm{d} \xi}|v(\xi)|^{2} \leq C_{1}|v(\xi)|^{2}+\sum_{j \neq 0} C_{1}|v(\xi+j)||v(\xi)| \leq C_{3}|v(\xi)|^{2}+\sum_{j \neq 0} C_{1}|v(\xi+j)|^{2}$.

Integrating this inequality over $[\xi, m+\varepsilon]$ with $\xi \in[m, m+\varepsilon]$ and $\varepsilon \leq 1 / 2$, we obtain

$$
\begin{aligned}
|v(\xi)|^{2} & \leq|v(m+\varepsilon)|^{2}+\int_{\xi}^{m+\varepsilon}\left(C_{3}|v(\zeta)|^{2}+\sum_{j \neq 0} C_{1}|v(\zeta+j)|^{2}\right) \mathrm{d} \zeta \\
& \leq|v(m+\varepsilon)|^{2}+\int_{\xi}^{m+\varepsilon}\left(C_{3}|v(\zeta)|^{2}+\sum_{j<0} C_{1}|\phi(\zeta+j)|^{2}\right) \mathrm{d} \zeta+C_{4}\|\phi\|_{L^{2}}^{2} \\
& \leq C(\varepsilon)\|\phi\|^{2}+\int_{\xi}^{m+\varepsilon} C_{3}|v(\zeta)|^{2} \mathrm{~d} \zeta
\end{aligned}
$$


where we used that $v(\xi+j)=\phi(\xi+j)$ for $j<0$ and exploited the estimates (3.8) and (3.9). Using Gronwall's inequality, we get

$$
\|v\|_{L^{2}([m, m+\varepsilon])}^{2} \leq \widetilde{C}\|\phi\|_{Y}^{2} .
$$

In summary, from (3.6) and (3.10), we finally conclude that

$$
\left\|v_{\xi}\right\|_{L^{2}([-m, m])} \leq K\|\phi\|_{Y}
$$

for all $\xi \geq 0$, where $v(\xi)$ is the solution of (3.7) with initial condition $\phi$ associated with a given $V_{0}=(\phi, \phi(0)) \in E_{j}^{\mathrm{s}}$. Note that the constant $K$ that appears in (3.11) does not depend on $j$ (and not on $V_{0}$ ). In addition, we have

$$
\left\|v_{\xi}\right\|_{H^{1}([-m, m])} \leq C K \mathrm{e}^{-\kappa \xi}\|\phi\|_{Y}
$$

for $\xi \geq m$ by using (3.7) and (3.6).

Having established these uniform estimates on $E_{j}^{\mathrm{s}}$, it remains to extend the semigroup $\Phi^{\mathrm{s}}(\xi)$ from $E_{j}^{\mathrm{s}}$ to the closure $\overline{E^{\mathrm{s}}}$, while maintaining the estimates (3.6). This can be done in a straightforward manner by approximating initial conditions in $\overline{E^{\mathrm{s}}}$ by elements in $E_{j}^{\mathrm{s}}$ in the $L^{2}$-sense and using compactness properties implied by the $H^{1}$-estimate (3.12) on any given bounded interval in $\xi$. We omit the details as they are similar (and in fact easier) than those given in [15].

\section{FREDHOLM PROPERTIES OF $\mathcal{T}$ IMPLY THE EXISTENCE OF DICHOTOMIES}

In this section, we prove that the non-autonomous equation (2.1) has an exponential dichotomy. The arguments are similar to those used in [18] in the case of modulated travelling waves. For this reason, we give an outline of the proof and provide details only where the arguments for forward-backward delay equations are different. The main strategy is to compare the non-autonomous operator with the constant-coefficient operator for which the existence of exponential dichotomies has been shown in the previous section.

After extending the operator $\mathcal{T}$ in Section 4.1 to a larger function space, we prove Theorem 1.1 in Sections 4.2 and 4.3, and Theorem 1.2 in Section 4.4.

4.1. The extension $S$ of $\mathcal{T}$. We consider the operator

$$
\mathcal{T}: \quad L^{2}(\mathbb{R}, Y) \rightarrow L^{2}(\mathbb{R}, Y), \quad V \longmapsto \frac{\mathrm{d} V}{\mathrm{~d} \xi}-\mathcal{A} V
$$

with domain $\mathrm{D}(\mathcal{T})$ given in (2.4). The adjoint $\mathcal{T}^{*}$ of $\mathcal{T}$ is also densely defined in $L^{2}(\mathbb{R}, Y)$ with domain $\mathrm{D}\left(\mathcal{T}^{*}\right)$ given in Lemma 2.3. Alternatively, we can consider $\mathcal{T}^{*}$ as a bounded operator, denoted by $\widehat{\mathcal{T}^{*}}$, from $\mathrm{D}\left(\mathcal{T}^{*}\right)$ to $L^{2}(\mathbb{R}, Y)$. Here, we 
consider $\mathrm{D}\left(\mathcal{T}^{*}\right)$ as a Banach space equipped with the graph norm. We denote by $S$ the adjoint operator $\left(\widehat{\mathcal{T}^{*}}\right)^{*}$ of $\widehat{\mathcal{T}^{*}}$, so that

$$
S: \quad L^{2}(\mathbb{R}, Y) \rightarrow \mathrm{D}\left(\mathcal{T}^{*}\right)^{*} .
$$

Note that $S$ restricted to $\mathrm{D}(\mathcal{T})$ coincides with $\mathcal{T}$. We remark that the notation $\left(\mathcal{T}^{*}\right)^{\text {ad }}$ instead of $S$ is used in [18].

By definition, the equation $S U=G$ means that $\left(\mathcal{T}^{*} W, U\right)=(W, G)$ for all $W \in \mathrm{D}\left(\mathcal{T}^{*}\right)$. The brackets denote the duality pairing of $\mathrm{D}\left(\mathcal{T}^{*}\right)$ and $\mathrm{D}\left(\mathcal{T}^{*}\right)^{*}$. In other words, $S U=G$ is a shortcut for

$$
-\int_{-\infty}^{\infty}\left\langle\partial_{\xi} W+\mathcal{A}(\xi)^{*} W, U\right\rangle_{Y} \mathrm{~d} \xi=\int_{-\infty}^{\infty}\langle W, G\rangle_{Y} \mathrm{~d} \xi, \quad \forall W \in \mathrm{D}\left(\mathcal{T}^{*}\right)
$$

where the scalar products $\langle\cdot, \cdot\rangle_{Y}$ are interpreted in the sense of distributions.

Lemma 4.1. Assume that $\mathcal{T}$ is Fredholm, then $S$ is also Fredholm with the same index. Furthermore, $\mathrm{N}(S)=\mathrm{N}(\mathcal{T})$.

Proof. The statements are consequences of [8, Section III $\S 5.5$ on p. 168] and [8, Section IV $\S 5.3$ on p. 236].

4.2. $\mathcal{T}$ is invertible. It is convenient to consider first the case that $\mathcal{T}$ is invertible before proceeding to the more general case that $\mathcal{T}$ is only Fredholm.

Lemma 4.2 ([18, Lemma 5.2]). Assume that $\mathcal{T}$ is invertible. For any $\xi_{0} \in$ $\mathbb{R}$ and any $G_{0} \in Y$, define $G(\xi, \eta):=G_{0}(\eta) \delta\left(\xi-\xi_{0}\right)$ where $\delta$ denotes the $\delta$ distribution. There is then a unique solution $U \in L^{2}(\mathbb{R}, Y)$ of the equation

$$
S U=G .
$$

The restrictions of $U$ to $\left(-\infty, \xi_{0}\right]$ and $\left[\xi_{0},+\infty\right)$ belong to $C^{0}\left(\left(-\infty, \xi_{0}\right], Y\right)$ and $C^{0}\left(\left[\xi_{0},+\infty\right), Y\right)$, respectively. The limits $U_{+}\left(\xi_{0}\right):=\lim \xi \backslash \xi_{0} U(\xi)$ and $U_{-}\left(\xi_{0}\right):=$ $\lim _{\xi} / \xi_{0} U(\xi)$ exist and satisfy the jump condition $U_{+}\left(\xi_{0}\right)-U_{-}\left(\xi_{0}\right)=G_{0}$. In addition, we have the estimate

$$
\|U\|_{L^{\infty}(\mathbb{R}, Y)}+\|U\|_{L^{2}(\mathbb{R}, Y)} \leq C\left|G_{0}\right|_{Y}
$$

where the constant $C$ is independent of $G_{0}$.

Proof. Note that the equation $S U=G$ is well-defined since $G \in \mathrm{D}(\mathcal{T})^{*}$ by Lemma 2.1. Without loss of generality, let $\xi_{0}=0$.

The proof is based on the comparison with an appropriate reference equation. Choose matrices $A_{j}^{\text {ref }}$ for $0 \leq|j| \leq m$ such that $\operatorname{det}\left(A_{-m}^{\text {ref }} A_{m}^{\text {ref }}\right) \neq 0$ and such that $\operatorname{det} \Delta(\mu) \neq 0$ for all $\mu \in \mathrm{i} \mathbb{R}$ where

$$
\Delta(\mu)=\sum_{j=-m}^{m} A_{j}^{\mathrm{ref}} \mathrm{e}^{j \mu}-\mu \mathrm{id} .
$$


Define

$$
\mathcal{A}_{\text {ref }}:\left(\begin{array}{c}
\phi \\
a
\end{array}\right) \longmapsto\left(\begin{array}{c}
\frac{\mathrm{d} \phi}{\mathrm{d} \eta} \\
A_{0}^{\mathrm{ref}} a+\sum_{1 \leq|j| \leq m} A_{j}^{\mathrm{ref}} \phi(j)
\end{array}\right)
$$

so that $\mathcal{A}_{0}$ is hyperbolic. We write $\mathcal{T}$ as

$$
\mathcal{T}=\mathcal{T}_{\text {ref }}+\mathcal{B}
$$

where $\mathcal{T}_{\text {ref }}=\frac{\mathrm{d}}{\mathrm{d} \xi}-\mathcal{A}_{\text {ref }}$ and

$$
\begin{aligned}
\mathcal{B}(\xi)=\mathcal{A}_{\mathrm{ref}}-\mathcal{A}(\xi) & : \\
\left(\begin{array}{l}
\phi \\
a
\end{array}\right) & \longmapsto\left(\left(A_{0}^{\mathrm{ref}}-A_{0}(\xi)\right) a+\sum_{1 \leq|j| \leq m}\left(A_{j}^{\mathrm{ref}}-A_{j}(\xi)\right) \phi(j)\right) .
\end{aligned}
$$

The strategy is to first seek a solution $V \in L^{2}(\mathbb{R}, Y)$ of the reference equation $S_{\text {ref }} V=G$ and afterwards a continuous solution $\widetilde{U}$ that satisfies $S \widetilde{U}=-\mathcal{B} V$. The sum $U:=V+\widetilde{U}$ then satisfies

$$
S U=S(V+\tilde{U})=S_{\text {ref }} V+\mathcal{B} V+S \tilde{U}=G
$$

as desired.

We begin by solving the equation

$$
S_{\text {ref }} V=\left(\frac{\mathrm{d}}{\mathrm{d} \xi}-\mathcal{A}_{\text {ref }}\right) V=G
$$

According to Proposition 3.1, the equation

$$
\frac{\mathrm{d} V}{\mathrm{~d} \xi}=\mathcal{A}_{\text {ref }} V
$$

has exponential dichotomies. Let $P_{\text {ref }}$ and (id $-P_{\text {ref }}$ ) be the corresponding projections such that (4.1) generates exponentially decaying $C^{0}$-semigroups on both $\mathrm{R}\left(P_{\text {ref }}\right)$ and $\mathrm{R}\left(\mathrm{id}-P_{\text {ref }}\right)$. For any $G_{0} \in Y^{1}$, define

$$
V(\xi):= \begin{cases}\mathrm{e}^{\mathcal{A}_{\text {ref }} P_{\text {ref }} \xi} P_{\text {ref }} G_{0} & \text { for } \xi>0 \\ -\mathrm{e}^{\mathcal{A}_{\text {ref }}\left(\text { id }-P_{\text {ref }}\right) \xi}\left(\text { id }-P_{\text {ref }}\right) G_{0} & \text { for } \xi<0 .\end{cases}
$$

By standard semigroup theory, the function $V$ is differentiable for $\xi \neq 0$ and satisfies (4.1) for $\xi \neq 0$. Furthermore, the limits $V_{+}^{0}:=\lim _{\xi \backslash 0} V(\xi)$ and $V_{-}^{0}:=$ 
1106 JÖrg HÄrTERICH. BJÖrn SANDSTEDE, AND ARnd SCHEEL

$\lim _{\xi>0} V(\xi)$ exist. For any test function $\chi \in C_{0}^{\infty}\left(\mathbb{R}, Y^{1}\right)$, we obtain

$$
\begin{aligned}
& -\int_{-\infty}^{\infty}\left\langle V, \frac{\mathrm{d} \chi}{\mathrm{d} \xi}+\mathcal{A}_{\mathrm{ref}}^{*} \chi\right\rangle_{Y} \mathrm{~d} \xi \\
& =-\int_{0}^{\infty}\left\langle\mathrm{e}^{\mathcal{A}_{\text {ref }} P_{\text {ref }} \xi} P_{\text {ref }} G_{0}, \frac{\mathrm{d} \chi}{\mathrm{d} \xi}+\mathcal{A}_{\mathrm{ref}}^{*} \chi\right\rangle_{Y} \mathrm{~d} \xi \\
& +\int_{-\infty}^{0}\left\langle\mathrm{e}^{\mathcal{A}_{\text {ref }}\left(\mathrm{id}-P_{\text {ref }}\right) \xi} P_{\text {ref }} G_{0}, \frac{\mathrm{d} \chi}{\mathrm{d} \xi}+\mathcal{A}_{\text {ref }}^{*} \chi\right\rangle_{Y} \mathrm{~d} \xi \\
& =\left.\left\langle\mathrm{e}^{\mathcal{A}_{\text {ref }} P_{\text {ref }} \xi} P_{\text {ref }} G_{0}, x\right\rangle_{Y}\right|_{\xi=0}+\left.\left\langle\mathrm{e}^{\mathcal{A}_{\text {ref }}\left(\text { id }-P_{\text {ref }}\right) \xi} P_{\text {ref }} G_{0}, x\right\rangle_{Y}\right|_{\xi=0} \\
& =V_{+}^{0}-V_{-}^{0}=\left\langle G_{0}, \chi(0)\right\rangle_{Y}=\int_{-\infty}^{\infty}\left\langle G_{0} \delta(\xi), \chi\right\rangle_{Y} \mathrm{~d} \xi
\end{aligned}
$$

using integration by parts and the fact that $V$ satisfies (4.1) for $\xi \neq 0$. This shows that, for $G_{0} \in Y^{1}, V$ satisfies $S_{\text {ref }} V=G$. For $G_{0} \in Y$, we define a solution $V \in L^{2}(\mathbb{R}, Y)$ of $S_{\text {ref }} V=G$ by approximating $G_{0}$ by a sequence in $Y^{1}$ and using the strong continuity of the semigroup (see [18, Section 5.3.1] for details).

In the next step, we solve

$$
S \tilde{U}=-\mathcal{B} V
$$

that involves the solution $V$ of the reference equation. The right-hand side $-\mathcal{B} V$ of (4.2) is in $L^{2}(\mathbb{R}, Y)$ since its first component vanishes completely, while the second component is continuous except at $\xi=0$ and decays exponentially as $\xi \rightarrow \pm \infty$. Since $S$ restricted to $\mathrm{D}(\mathcal{T})$ coincides with $\mathcal{T}$, and since both operators are invertible by assumption and by Lemma 4.1, we can solve (4.2) and obtain a unique solution $\widetilde{U}=(\tilde{\phi}, \tilde{a}) \in \mathrm{D}(\mathcal{T})$. Using the fact that the first component of $-\mathcal{B} V$ vanishes, we see that $\tilde{\phi}(\xi, \eta)=\tilde{a}(\xi+\eta)$ with $\tilde{a} \in H^{1}\left(\mathbb{R}, \mathbb{C}^{n}\right)$. In particular, $\tilde{U}$ is continuous on $\mathbb{R}$ with values in $Y$ and

$$
\|\tilde{U}\|_{H^{1}(\mathbb{R}, Y)}+\|\tilde{U}\|_{L^{2}\left(\mathbb{R}, Y^{1}\right)} \leq C\|V\|_{L^{2}(\mathbb{R}, Y)} .
$$

Lastly, as mentioned earlier, the sum $U=\widetilde{U}+V$ satisfies all the properties stated in the lemma. In particular, it is continuous on $\mathbb{R}^{-}$and $\mathbb{R}^{+}$, and the jump at $\xi=0$ is exactly the jump of $V$ at $\xi=0$. Therefore, $U_{+}(0)-U_{-}(0)=G_{0}$.

The previous lemma allows us to define a continuous, injective map

$$
\Pi\left(\xi_{0}\right): Y \longrightarrow Y \times Y, G_{0} \longmapsto\left(U_{+}\left(\xi_{0}\right), U_{-}\left(\xi_{0}\right)\right) .
$$

Using the canonical projections $P_{i}\left(\xi_{0}\right): Y \times Y \rightarrow Y$ defined by $P_{i}\left(\xi_{0}\right)\left(U_{1}, U_{2}\right)=$ $U_{i}$ for $i=1,2$, we have the relation

$$
G_{0}=P_{1}\left(\xi_{0}\right) \Pi\left(\xi_{0}\right) G_{0}-P_{2}\left(\xi_{0}\right) \Pi\left(\xi_{0}\right) G_{0}
$$


for any $G_{0} \in Y$.

Lemma 4.3 ([18, Lemma 5.3]). Suppose that $\mathcal{T}$ is invertible. The images $\mathrm{R}\left(P_{i}\left(\xi_{0}\right) \Pi\left(\xi_{0}\right)\right)$ are then closed subspaces, and we have $\mathrm{R}\left(P_{1}\left(\xi_{0}\right) \Pi\left(\xi_{0}\right)\right) \oplus$ $\mathrm{R}\left(P_{2}\left(\xi_{0}\right) \Pi\left(\xi_{0}\right)\right)=Y$ for each $\xi_{0} \in \mathbb{R}$.

We can then define a family of projections $P(\xi)$ such that $\mathrm{R}(P(\xi))=$ $\mathrm{R}\left(P_{1}(\xi) \Pi(\xi)\right)$ and $\mathrm{N}(P(\xi))=\mathrm{R}\left(P_{2}(\xi) \Pi(\xi)\right)$. Note that, for any $U_{+} \in \mathrm{R}\left(P\left(\xi_{0}\right)\right)$, there exists a unique strong solution $V^{\mathrm{s}}(\xi)$ of (2.2), defined for $\xi>\xi_{0}$, with initial value $V^{\mathrm{s}}\left(\xi_{0}\right)=U_{+}$. Analogously, for any $U_{-} \in \mathrm{N}\left(P\left(\xi_{0}\right)\right)$, there exists a unique strong solution $V^{\mathrm{u}}(\xi)$ of $(2.2)$, defined for $\xi<\xi_{0}$, with initial value $V^{\mathrm{u}}\left(\xi_{0}\right)=U_{-}$. This projections define the desired exponential dichotomies.

Lemma 4.4 ([18, Lemma 5.5]). The family $P(\xi)$ of projections together with the solutions $V^{\mathrm{s}}$ and $V^{\mathrm{u}}$ defines an exponential dichotomy on $\mathbb{R}$.

4.3. $\mathcal{T}$ is Fredholm. Again, we closely follow the proof given in [18] for parabolic equations in cylinders. Since most of the details are identical, save for notation, we comment only on those two parts of the proof where the arguments in [18, Section 5.3.2] need to be modified. First, the proof given in [18, Section 5.3.2] uses the fact that the null spaces of $\mathcal{T}$ and $S$ coincide. We proved in Lemma 4.1 that this is always the case. Second, the following weak uniqueness condition (U1) has been used in [18].

(U1) If $V$ is in the null space of $\mathcal{T}$ or the adjoint operator $\mathcal{T}^{*}$ and $V\left(\xi_{0}\right)=0$ for some $\xi_{0}$, then $V$ vanishes identically.

This assumption is, however, equivalent to Hypothesis 1.1 on account of Lemma 2.2. Now that the above two properties are established, the abstract proof in [18] applies to our situation and gives the existence of exponential dichotomies. This completes the proof of Theorem 1.1 on the existence of exponential dichotomies.

4.4. Proof of Theorem 1.2. It suffices to show that the hypotheses of Theorem 1.2 imply the hypotheses of Theorem 1.1 which we proved in the last section. Proposition 2.1 which is due to Mallet-Paret [11] implies that $\mathcal{L}$ is Fredholm provided it is asymptotically hyperbolic. We established in Lemma 2.4 that $\mathcal{T}$ is Fredholm whenever $\mathcal{L}$ is. It therefore remains to demonstrate that Hypothesis 1.1 is a consequence of the following Hypothesis 4.1 which we assumed in Theorem 1.2.

Hypothesis 4.1. Assume that neither $\operatorname{det} A_{m}(\xi)$ nor $\operatorname{det} A_{-m}(\xi)$ vanish on any open interval.

To establish that Hypothesis 1.1 follows from Hypothesis 4.1, suppose that $a \in \mathrm{N}(\mathcal{L})$ such that $a(\xi)$ vanishes identically on the interval $\left[\xi_{0}-m, \xi_{0}+m\right]$. We want to prove that this implies that $a(\xi)=0$ for all $\xi \in \mathbb{R}$ and argue by contradiction. Note that, by Lemma 2.2, $a$ is a classical solution of (2.1). Thus, without loss of generality, we assume that

$$
\xi_{1}:=\inf \left\{\xi \geq \xi_{0}: a(\xi+m) \neq 0\right\}
$$




\section{JÖrG HÄrTERICH. BJÖRn SANDSTEDE, AND ARnd SCHEEL}

exists and is finite. Since $a(\xi+j)=0$ for $|j| \leq m$ and $\xi_{0} \leq \xi \leq \xi_{1}$, we have

$$
a^{\prime}(\xi)=\sum_{j=-m}^{m} A_{j}(\xi) a(\xi+j)=A_{m}(\xi) a(\xi+m)
$$

for all $\xi \in\left[\xi_{1}, \xi_{1}+1\right)$. By the definition of $\xi_{1}$, there exists an open and nonempty interval $J \subset\left(\xi_{1}, \xi_{1}+1\right)$ such that $a(\xi+m) \neq 0$ for any $\xi \in J$. Thus, we conclude that

$$
a^{\prime}(\xi)=A_{m}(\xi) a(\xi+m) \neq 0
$$

for some $\xi \in J$, since $\operatorname{det} A_{m}(\xi)$ does not vanish identically on $J$ by Hypothesis 4.1. This contradicts the definition of $\xi_{1}$.

\section{REFERENCES}

[1] R. Bellman And K. CoOKe, Differential difference equations. Academic Press, New York, 1963.

[2] S. BENZONI-GAVAGE, Stability of semi-discrete shock profiles by means of an Evans function in infinite dimensions, J. Dynam. Diff. Eqns. (to appear).

[3] R.P. BOAS, Entire functions. Academic Press, New York, 1954.

[4] W.A. Coppel, Dichotomies in stability theory. Lecture Notes in Mathematics 629, Springer, Berlin, 1978.

[5] R.J. Duffin AND A.C. SCHAEFFER, Some properties of functions of exponential type, Bull. Amer. Math. Soc.44 (1938), 236-240.

[6] J.K. Hale AND X.-B. LIN, Heteroclinic orbits for retarded functional differential equations, J. Diff. Eqns. 65 (1986), 175-202.

[7] D. HENRY, Geometric theory of semilinear parabolic equations. Lecture Notes in Mathematics 804, Springer, New York, 1981.

[8] T. KATO, Perturbation theory for linear operators. Springer, Berlin, 1976.

[9] X.-B. LIN, Using Melnikov's method to solve Shilnikov's problems, Proc. Roy. Soc. Edinburgh 116A (1990), 295-325.

[10] G.J. Lord, D. Peterhof, B. Sandstede, And A. Scheel, Numerical computation of solitary waves on infinite cylinders, SIAM J. Numer. Anal. 37 (2000), 1420-1454.

[11] J. MALLET-PARET, The Fredholm alternative for functional differential equations of mixed type, J. Dynam. Diff. Eqns. 11 (1999), 1-47.

[12] J. Mallet-Paret and S.M. Verduyn Lunel, Exponential dichotomies and Wiener-Hopf factorizations for mixed-type functional differential equations, J. Diff. Eqns. (to appear).

[13] A. PAZY, Semigroups of linear operators and applications to partial differential equations. Springer, Berlin, 1983.

[14] D. PETERHOF, B. SANDSTEDE AND A. SCHEel, Exponential dichotomies for solitary waves of semilinear elliptic equations on infinite cylinders, J. Diff. Eqns. 140 (1997), 266-308.

[15] A. RUSTICHINI, Functional differential equations of mixed type: the linear autonomous case, J. Dynam. Diff. Eqns. 1 (1989), 121-143.

[16] B. SANDSTEDE, Verzweigungstheorie homokliner Verdopplungen. Ph.D. Thesis, University of Stuttgart, 1993. 
[17] B. SAndstede, Stability of travelling waves. In: "Handbook of Dynamical Systems II" (B. Fiedler, ed.). Elsevier, (2002) 983-1055.

[18] B. SANDSTEDE AND A. SCHEel, On the structure of spectra of modulated travelling waves, Math. Nachr. 232 (2001), 39-93.

[19] S.M. VERDUYN LUNEL, About completeness for a class of unbounded operators, J. Diff. Eqns. 120 (1995), 108-132.

JÖRG HÄRTERICH :

Institut für Mathematik I

Freie Universität Berlin

Arnimallee 2-6

14195 Berlin, Germany

BJÖRN SANDSTEDE :

Department of Mathematics

The Ohio State University

231 West 18th Avenue

Columbus, OH 43210, U.S.A.

ARND SCHEEL :

School of Mathematics

University of Minnesota

206 Church Street SE

Minneapolis, MN 55455, U.S.A.

Received: June 12th, 2001. 